\title{
Horseshoes and Arnold Diffusion for Hamiltonian Systems on Lie Groups
}

\section{PHILIP J. HOLMES \& JERROLD E. MARSDEN}

\section{\$1. Introduction}

This paper presents theorems which establish the existence of horseshoes and Arnold diffusion for nearly integrable Hamiltonian systems associated with Lie groups. The methods are based on our two previous papers, Holmes and Marsden [1982a], [1982b]. The two main examples treated here are as follows:

1. A simplified model of the rigid body with attachments. This system has horseshoes (with one attachment) and Arnold diffusion (with two or more attachments).

2. A rigid body under gravity, close to a symmetric (Lagrange) top. This system is shown to have horseshoes (and hence is not integrable).

The main new feature here is the presence of Lie groups. Both the symmetry groups and the basic phase spaces involve Lie groups and our perturbation methods must be modified to take this into account. As in our previous work, the results hinge on reduction together with a method of Melnikov. This is used to analyze the perturbation of a homoclinic orbit in an integrable Hamiltonian system. In the first example the unperturbed system is the free rigid body which has a homoclinic orbit lying on a sphere. This sphere arises as the coadjoint orbit for the rotation group SO (3), and the computation of Poisson brackets needed in the Melnikov theory is most easily done using the (Kirillov, Arnold, Kostant and Souriau) theory of coadjoint orbits and the Lie-Poisson bracket on the dual of a Lie algebra. This theory is reviewed in Section 2. Reduction in the sense of Marsden and Weinstein [1974] shows that the phase space for a rigid body under gravity is $T^{*} S^{2}$, the cotangent bundle of a sphere. This and its connection with Euler angles and coadjoint orbits in the Euclidean group is explained in Section 3. This section thus sets up the basic phase spaces needed in the analysis of our second example.

Section 4 develops the Melnikov theory when the phase space is a product of the dual of a Lie algebra and a set of action angles variables. This is applied to a model problem based on the rigid body with attachments in Section 5 .

Section 6 develops the Melnikov theory for systems on a phase space where the unperturbed system admits an $S^{1}$ symmetry and has a homoclinic orbit in the 
reduced phase space. This generalization does not assume the original phase space is a product. This generalization is needed for and is applied to the nearly symmetric heavy top in Section 7. It is also needed for a full treatment of the rigid body with attachments given by Koiller [1982].

The two examples presented here were selected because of their physical interest and because they provide good models for how the Melnikov theory must be modified for systems with topologically nontrivial phase spaces.

The presence of horseshoes in the motion of a nearly symmetry heavy top implies, among other things, that the dynamics is complex and cannot be captured by averaging methods (cf. Akulenko, Leshchenko and Chernouśko [1979]), that the dynamics has periodic orbits of arbitrarily high period embedded in an invariant Cantor set and that the system admits no additional analytic integrals. The latter fact is consistent with known classical results, but the existence of horseshoes is a stronger and, we think, more significant assertion. Ziglin [1981] recently outlined a general nonintegrability theorem that includes the nonstandard rigid body cases but his proof proceeds along different lines and does not seem to yield horseshoes. Some numerical work for the heavy top is given in Galgoni, Giorgilli and Strelcyn [1981].

We expect that dissipative and forcing terms added to these systems can be dealt with along the lines of Holmes and Marsden [1982a]

Acknowledgements. We thank Allan Kaufman, Jair Koiller, Tudor Ratiu, Steve Smale, Shlomo Sternberg, and Alan Weinstein for motivation and several useful comments.

\section{§2. Hamiltonian Systems on Lie Groups and Semi-Direct Products}

Since the basic paper of Arnold [1966] Lie groups have played an important role in the construction of phase spaces and the symmetry properties of some important mechanical systems. For systems such as the rigid body one wishes to realize the classical Euler equations as Hamiltonian equations on an appropriate phase space to apply Hamiltonian perturbation techniques. For a rigid body free to rotate about its center of mass, the basic phase space is $T^{*} S O(3)$ which is conveniently parametrized by the Euler angles $(\phi, \psi, \theta)$ and the corresponding conjugate momenta $\left(p_{\phi}, p_{\psi}, p_{\theta}\right)$. However the $S O(3)$ symmetry of the problem enables one to reduce this three degree of freedom problem to a one degree of freedom system whose phase space is a sphere in body-angular momentum space.

For purposes of this paper we are interested mostly in the Poisson bracket structure since it is this which directly enters the Melnikov theory. In what follows we describe the Poisson structure associated with a Lie group and its connection with coadjoint orbits. (For the same theory with emphasis on the symplectic geometry, see Abraham and Marsden [1978, Chapter 4].) Proofs may be readily supplied by referring to the preceding reference, to Arnold [1978] and to Guillemin and Sternberg [1980]. 
(a) Generalities on Lie-Poisson structures. Let $G$ be a Lie group and $g$ its Lie algebra. For $\xi, \eta \in g,[\xi, \eta]$ denotes the Lie bracket of $\xi$ and $\eta$. Let $\mathrm{g}^{*}$ denote the dual space of $\mathrm{g}$. For $F: \mathrm{g}^{*} \rightarrow \mathbf{R}$, and the variable in $\mathrm{g}^{*}$ denoted by $\mu$, define $\delta F / \delta \mu: \mathfrak{g}^{*} \rightarrow \mathfrak{g}$ by

$$
D F(\mu) \cdot v=\left\langle\nu, \frac{\delta F}{\delta \mu}\right\rangle,
$$

where $\langle$,$\rangle denotes the pairing between \mathfrak{g}^{*}$ and $\mathfrak{g}$; and $D F(\mu): \mathfrak{g}^{*} \rightarrow \mathbf{R}$ is the usual (Frechét) derivative. It is understood that $\delta F / \delta \mu$ is evaluated at the point $\mu$.

The Lie-Poisson bracket of two functions $F, G: \mathfrak{g}^{*} \rightarrow \mathbf{R}$ is defined by

$$
\{\{F, G\}\}(\mu)=-\left\langle\mu,\left[\frac{\delta F}{\delta \mu}, \frac{\delta G}{\delta \mu}\right]\right\rangle .
$$

This bracket makes the smooth functions from $\mathrm{g}^{*}$ to $\mathbf{R}$ into a Lie algebra. (The only nonobvious condition is Jacobi's identity.) The bracket (2.2) was introduced (for finite-dimensional Lie algebras) by Lie in 1887 in Volume 2 of his treatise on transformation groups. [We thank Alan Weinstein for pointing out the history of this bracket.]

Next we describe the relationship between the Lie-Poisson bracket and coadjoint orbits. For $g \in G$, let $\mathrm{Ad}_{g}: \mathfrak{g} \rightarrow \mathfrak{g}$ be the adjoint representation (the linearization of the map $I_{g}: h \mapsto g h g^{-1}$ at $h=$ identity) and $\operatorname{Ad}_{g^{-1}}^{*}: \mathfrak{g}^{*} \rightarrow \mathrm{g}^{*}$ the coadjoint representation. For $\mu_{0} \in g^{*}$, let $O=\left\{\operatorname{Ad}_{g}^{*-1} \mu_{0}: g \in G\right\}$ be the orbit of $\mu_{0}$. A theorem of Kirillov, Kostant and Souriau states that $\mathscr{O}$ is a symplectic manifold. We now describe the symplectic structure. For $\mu \in \mathcal{O}$, tangent vectors to 0 at $\mu$ have the following form: let $\xi \in g$ and define $\bar{\xi}(\mu) \in g^{*}$ by $\eta \mapsto\langle\mu,[\xi, \eta]\rangle$. Then $\tilde{\xi}(\mu)$ is tangent to 0 at $\mu$. The formula

$$
\omega_{\mu}(\tilde{\xi}(\mu), \tilde{\eta}(\mu))=-\langle\mu,[\xi, \eta]\rangle
$$

defines a symplectic form on 0 . Formulas (2.2) and (2.3) are related as follows: for $F, G: \mathfrak{g}^{*} \rightarrow \mathbf{R}$,

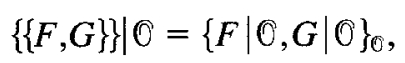

where \{\}$_{\odot}$ is the Poisson bracket computed from the symplectic structure (2.3) on 0 .

If $H: \mathrm{g}^{*} \rightarrow \mathbf{R}$ is a given Hamiltonian, there is a unique vector field $X_{H}$ on $\mathrm{g}^{*}$ such that any function $F: \mathrm{g}^{*} \rightarrow \mathbf{R}$ which evolves along the flow of $X_{H}$ satisfies

$$
\dot{F}=\{\{F, H\}\} \text {. }
$$

Explicit equations of motion for $\mu \in \mathfrak{g}^{*}$ can be computed directly from (2.5) and (2.2) in examples, without the computation of coadjoint orbits, by letting $F$ be coordinate functions on $\mathfrak{g}^{*}$. The equations so obtained are thus

$$
\dot{\mu}=X_{H}(\mu) \text {. }
$$


The vector field $X_{H}$ is necessarily tangent to the coadjoint orbits; thus if $\mu(0) \in$ 0 , then $\mu(t) \in \mathcal{O}$ as well. Furthermore, $X_{H} \mid \mathcal{O}=X_{H \mid \mathcal{0}}$ where $X_{H \mid \mathcal{O}}$ is the Hamiltonian vector field on $\mathrm{O}$ computed from $H$ using the symplectic structure (2.3).

As we shall see in the next sections, the classical Euler equations for a rigid body (with or without gravity) can be expressed in the form (2.5).

Remark. In Arnold [1966] and Ebin and Marsden [1970] it is shown that the equations of an incompressible fluid also fall into this class using the group $\mathscr{D}_{\text {vol }}$ of volume preserving diffeomorphisms of space. For compressible flow, the appropriate group is the semi-direct product of diffeomorphisms and functions. (The reason semi-direct products are relevant is explained abstractly below.) When appropriately coupled to the electromagnetic field, equations (2.5) also include the equations of plasma physics and magnetohydrodynamics (see Marsden and Weinstein [1982]).

For the rigid body free to rotate about a fixed point, the basic phase space one starts with is $T^{*} S O(3)$, irrespective of whether gravity is present. In the absence of gravity, reduction by $S O(3)$ leads naturally to the Lie-Poisson structure for the Lie algebra of $S O(3)$. However when gravity is present, reduction by $S^{1}$ leads to the Lie-Poisson structure for the Lie algebra of the Euclidean group; i.e. the semidirect product $S O(3) \times \mathbf{R}^{3}$. Proofs of assertions made in the following may be found in one or more of Abraham and Marsden [1978], Guillemin and Sternberg [1980] and Ratiu and van Moerbeke [1982].

(b) Lie-Poisson structures for reduction by a subgroup. [The heavy rigid top is done two separate ways in Section 7, namely in terms of Euler angles and in terms of the Lie-Poisson bracket. Those who wish only to read the Euler angle proof may omit the rest of this section and the third part (c) of the next section.]

Let $G$ be a Lie group and $T^{*} G$ its cotangent bundle. Let $\nu \in \mathfrak{g}^{*}$ and let $G_{v}=\left\{g \in G: \operatorname{Ad}_{g^{-1}}^{*} \nu=v\right\}$ be the isotropy subgroup of $\nu$. Now $G_{v}$ acts on $G$ by left translation and hence on $T^{*} G$. This action has an $\mathrm{Ad}^{*}$-equivariant momentum map

$$
J^{v}: T^{*} G \rightarrow \mathfrak{g}^{*}
$$

where $\mathfrak{g}_{\nu}$ is the Lie algebra of $G_{\nu}$. In fact, for $\alpha_{g} \in T_{g}^{*} G$ and $\xi \in \mathfrak{g}_{v}$,

$$
\left\langle J^{v}\left(\alpha_{g}\right), \xi\right\rangle=\left\langle\alpha_{g}, T_{e} R_{g} \cdot \xi\right\rangle
$$

where $R_{g}: G \rightarrow G$ denotes right translation by $g$ and $\langle$,$\rangle denotes the pairings$ between $\mathrm{g}_{v}^{*}$ and $\mathrm{g}_{v}$ or $T_{g}^{*} G$ and $T_{g} G$ as appropriate.

Now let $\mu \in \mathrm{g}^{*}$ and $\bar{\mu} \in \mathrm{g}_{v}^{*}$ be its restriction to $\mathfrak{g}_{v}$. We shall henceforth make the (generic) assumption that $G_{v}$ is abelian. [Sternberg and Ratiu have pointed out that this assumption is not necessary; however it is true in cases of interest in this paper, so we shall not attempt to explain the general case here; see Ratiu [1982], Guillemin and Sternberg [1982] and Marsden, Ratiu and Weinstein [1982].] Then the reduced space of $T^{*} G$ relative to the action of $G_{v}$ is 


$$
P_{\bar{\mu}, \nu}=\left(J^{\nu}\right)^{-1}(\bar{\mu}) / G_{\nu} .
$$

Let $\langle\langle\rangle$,$\rangle be a left invariant metric on G$ and $K: T^{*} G \rightarrow \mathbf{R}$ the corresponding kinetic energy function: $K\left(\alpha_{g}\right)=(1 / 2)\left\langle\left\langle\alpha_{g}, \alpha_{g}\right\rangle\right\rangle$. Let $\alpha_{\mu}$ be the one form on $G$ defined at $g \in G$ by minimizing $K$ over the affine space of $\alpha_{g}$ such that $J^{\nu}\left(\alpha_{g}\right)=\bar{\mu}$.

Remark. If $G_{v}=S^{1}$ and $\xi \in \mathfrak{g}_{v}$ is the element corresponding to $\bar{\mu}$ via $\langle\langle\rangle$, i.e. $\langle\langle\xi, \eta\rangle\rangle=\bar{\mu}(\eta)$ for all $\eta \in g_{v}$, then for $v \in T_{g} G$,

$$
\alpha_{\mu}(g) \cdot \mathrm{v}=\left\langle\left\langle T_{e} R_{g} \xi, \mathrm{v}\right\rangle\right\rangle \cdot \frac{\langle\langle\xi, \xi\rangle\rangle}{\left\langle\left\langle\mathrm{Ad}_{\mathrm{g}^{-1}} \xi, \mathrm{Ad}_{g^{-1}} \xi\right\rangle\right\rangle} .
$$

The function $K\left(\alpha_{\mu}(g)\right)=V_{\mu}(g)$ is called the amended potential.

The map $\alpha_{q} \mapsto \alpha_{q}-\alpha_{\mu}$ induces a symplectic diffeomorphism of $P_{\bar{\mu}, v}$ with $T^{*}\left(G / G_{v}\right)$ by Theorem 4.3.3 of Abraham and Marsden [1978].

Remark. In general, the symplectic form on $T^{*}\left(G / G_{v}\right)$ is the canonical one plus a "magnetic" field $\Omega_{\mu}$. (See Kummer [1981] for the interpretation of the class of $\Omega_{\mu}$ as a Chern class.) For the examples in this paper $\Omega_{\mu} \neq 0$. It is clear that $\alpha_{\mu} \neq 0$ and it is necessary to use it to form the amended potential. If this is added to the standard potential for the heavy top, we recover the effective potential. As we shall see below, this agrees with that in standard texts (Goldstein [1980, formula 5-60, page 215]) and is a special case of Theorem 4.5.6 of Abraham and Marsden [1978].

Now consider the semi-direct product $G \times \mathfrak{g}$ of $G$ with the additive group $\mathfrak{g}$ with $G$ acting on $\mathfrak{g}$ by the adjoint action. For $v \in \mathfrak{g}^{*}$, let

$$
\begin{gathered}
f^{\nu}: G \times g \rightarrow \mathbf{R} \\
f^{v}(g, \xi)=\left\langle\nu, \operatorname{Ad}_{g} \xi\right\rangle,
\end{gathered}
$$

i.e. $f(g, \cdot)=\operatorname{Ad}_{g}^{*} \nu$. Write $f_{\xi}^{\nu}(g)=f^{\nu}(g, \xi)$. From the identity

$$
f^{v}\left(g_{1}^{-1} g, \xi\right)=f^{v}\left(g, \operatorname{Ad}_{g_{1}} \xi\right)
$$

we see that $G \times g$ acts on $T^{*} G$ by

$$
(g, \xi) \cdot \alpha_{h}=t_{d f_{\xi}^{v}} \cdot\left(T L_{g}\right)^{*} \cdot \alpha_{h},
$$

where $t_{d f_{\xi}^{v}}$ is fiber translation in $T^{*} G$ by the differential of $f_{\xi}^{v}$. The action (2.12) is symplectic and has an $\mathrm{Ad}^{*}$-equivariant momentum map

$$
\Lambda^{\nu}: T^{*} G \rightarrow \mathrm{g}^{*} \times \mathrm{g}^{*}
$$

given by

$$
\Lambda^{v}\left(\alpha_{g}\right)=\left(\left(T_{e} L_{g^{-1}}\right)^{*} \alpha_{g}, \operatorname{Ad}_{g}^{*} v\right)
$$

we shall write $m=\left(T_{e} L_{g^{-1}}\right)^{*} \alpha_{g}$ and $\mathrm{v}=\operatorname{Ad}_{g}^{*} \nu$. (See Abraham and Marsden 
[1978, Exercise 4.2C] and Guillemin and Sternberg [1980].) We note that the adjoint action of $G \times \mathfrak{g}$ on $\mathfrak{g} \times \mathfrak{g}$ is

$$
\operatorname{Ad}_{(g, \xi)}(\eta, \check{\zeta})=\left(\operatorname{Ad}_{g} \eta, \operatorname{Ad}_{g} \zeta+\left[\xi, \operatorname{Ad}_{g} \eta\right]\right),
$$

and that the Lie bracket on $\mathfrak{g} \times \mathfrak{g}$ is

$$
\left[\left(\xi_{1}, \eta_{1}\right),\left(\xi_{2}, \eta_{2}\right)\right]=\left(\left[\xi_{1}, \xi_{2}\right],\left[\xi_{1}, \eta_{2}\right]+\left[\eta_{1}, \xi_{2}\right]\right) .
$$

One can check directly from (2.14) and (2.12) that $\Lambda^{\nu}$ is equivariant. (The identity

$$
\operatorname{Ad}_{g}[\xi, \eta]=\left[\operatorname{Ad}_{g} \xi, \operatorname{Ad}_{g} \eta\right]
$$

is useful in verifying this.)

Observe that $\Lambda^{v}$ is invariant under the left action of $G_{v}$; i.e. for $h \in G_{v}$

$$
\Lambda^{v}\left(T L_{h^{-1}}^{*} \alpha_{g}\right)=\Lambda^{v}\left(\alpha_{g}\right) \text {. }
$$

Thus, $\Lambda^{v}$ induces a map

$$
\bar{\Lambda}^{\nu}: P_{\bar{\mu}, \nu} \rightarrow \mathrm{g}^{*} \times \mathrm{g}^{*} .
$$

One can check that $\bar{\Lambda}^{v}$ is a diffeomorphism of $P_{\bar{\mu}, \nu}$ onto the orbit $\mathcal{O}_{\mu, \nu}$.

2.1 Theorem. $\bar{\Lambda}^{v}: P_{\bar{\mu}, \nu} \rightarrow \mathcal{O}_{\mu, \nu}$ is a symplectic diffeomorphism.

Remarks. 1. This result is due to Ratiu [1981], [1982]. The proof we give is motivated by Guillemin and Sternberg [1980].

2. For the Lagrange top, 2.1 can be proved by a direct, but messy, calculation which we outline in the next section.

3. See Guillemin and Sternberg [1982] for an alternative proof using Proposition 1.2 of Kazhdan, Kostant and Sternberg [1978].

4. The result may be generalized to the case where $\mathfrak{g} \times \mathfrak{g}$ is replaced by a semidirect product $g \times V$. See Ratiu [1982], Guillemin and Sternberg [1982], and Marsden, Ratiu and Weinstein [1982] for details.

Proof of Theorem 2.1. Since the symplectic form on $P_{\bar{\mu}, \nu}$ is induced from the canonical symplectic structure on $T^{*} G$ and that on $0_{\mu, v}$ is determined by the LiePoisson bracket, it suffices to show that $\Lambda^{v}$ commutes with Poisson brackets. This, however, is a general fact about $\mathrm{Ad}^{*}$-equivariant momentum maps and collective Hamiltonians proved in the next two lemmas.

2.2 Lemma. Let $J: P \rightarrow \mathfrak{1}^{*}$ be an $\mathrm{Ad}^{*}$-equivariant momentum map for the right action of a Lie group $K$ on the symplectic manifold $P$. Let $F: f^{*} \rightarrow \mathbf{R}$. Then the Hamiltonian vector field $X_{F \circ}$ for the (collective) Hamiltonian $F \circ J: P \rightarrow \mathbf{R}$ is given at $x \in P$ by

$$
X_{F o J}(x)=\left(\frac{\delta F}{\delta \mu}\right)_{P}(x)
$$


where $\delta F / \delta \mu \in \mathfrak{t}$ is evaluated at $\mu=J(x)$ and $\xi_{P}$ stands for the vector field on $P$ generated by the Lie algebra element $\xi$.

Proof. By the chain rule and definition of the functional derivative,

$$
\begin{aligned}
d(F \circ J) \cdot \mathrm{v}_{x} & =d F(\mu) \cdot d J(x) \cdot \mathrm{v}_{x} \\
& =\left\langle d J(x) \cdot \mathrm{v}_{x}, \frac{\delta F}{\delta \mu}\right\rangle
\end{aligned}
$$

where $\mathrm{v}_{x} \in T_{x} P$ and $\mu=J(x)$ as above. By definition of a momentum map,

$$
\left\langle d J(x) \cdot \mathrm{v}_{x}, \frac{\delta F}{\delta \mu}\right\rangle=\omega_{x}\left(\left(\frac{\delta F}{\delta \mu}\right)_{P}, \mathrm{v}_{x}\right)
$$

where $\omega$ is the symplectic form. Thus

$$
d(F \circ J) \cdot \mathrm{v}_{x}=\omega_{x}\left(\left(\frac{\delta F}{\delta \mu}\right)_{P}, \mathrm{v}_{x}\right)^{\bullet}
$$

which means $X_{F \circ J}=(\delta F / \delta \mu)_{P}$.

2.3 Lemma. Under the assumptions of the preceding lemma, if $F, G: \mathfrak{f}^{*} \rightarrow$ $\mathbf{R}$, then

$$
\{F \circ J, G \circ J\}=\{\{F, G\}\} \circ J
$$

Proof.

$$
\{F \circ J, G \circ J\}=\omega_{x}\left(\left(\frac{\delta F}{\delta \mu}\right)_{P},\left(\frac{\delta F}{\delta \mu}\right)_{P}\right)
$$

by definition of Poisson brackets on $P$ and the preceding lemma. On the other hand, the Lie-Poisson bracket is

$$
\{\{F, G\}\}(\mu)=-\left\langle J(x),\left[\frac{\delta F}{\delta \mu}, \frac{\delta G}{\delta \mu}\right]\right\rangle .
$$

Moreover, Ad*-equivariance gives (Abraham and Marsden [1978, Corollary 4.2.9])

$$
\left\langle J(x),\left[\frac{\delta F}{\delta \mu}, \frac{\delta F}{\delta \mu}\right]\right\rangle=-\left\{\left\langle J(x), \frac{\delta F}{\delta \mu}\right\rangle,\left\langle J(x), \frac{\delta F}{\delta \mu}\right\rangle\right\}
$$

in which $\mu$ is fixed and $x$ is variable. There is a minus sign here since $J$ is generated by a right action. Holding $\delta F / \delta \mu$ fixed,

$$
d\left\langle J(x), \frac{\delta F}{\delta \mu}\right\rangle \cdot \mathrm{v}_{x}=\left\langle d J(x) \cdot \mathrm{v}_{x}, \frac{\delta F}{\delta \mu}\right\rangle
$$


so $\langle J(x), \delta F / \delta \mu\rangle$ generates the same Hamiltonian vector field as $F \circ J$, as in 2.2 . Thus, substitution of (2.21) in (2.20) yields (2.19).

Remarks. As we shall see, the heavy rigid top Hamiltonian is a collective Hamiltonian for $J^{v}$. This is compatible with the reduction picture: a Hamiltonian system on the reduced space $P_{\bar{\mu}, \nu}$ can be written in the form

$$
\dot{F}=\{\{F, H\}\}
$$

where $H$ is written in terms of the variables $(m, \mathrm{v}) \in \mathrm{g}^{*} \times \mathrm{g}^{*}$.

\section{\$3. The Rigid Body}

This section is divided into three parts. The first part explains how to write the equations of a rigid body free to rotate about its center of mass in Lie-Poisson form (2.5). The second part recalls the Euler angle formulation of the heavy top and the third part puts it into Lie-Poisson form giving the explicit relationships with Euler angles. [The third part may be omitted if desired since we give two proofs of our main result for the heavy top, one using only Euler angles and the other using the Lie-Poisson bracket.]

(a) The free rigid body. The free rigid body is a left invariant Hamiltonian system on $T^{*} S O(3)$, where $S O(3)$ is the group of proper orthogonal linear transformations of $\mathbf{R}^{3}$ to itself. By general facts about reduction we know that the equations of motion must be in the form (2.5); this is true of any left invariant Hamiltonian system on a Lie group $G$. For the free rigid body we can bypass the Euler angle description (the relevant formulas are given in Table 1 below).

The Lie algebra $s o(3)$ of $S O(3)$ consists of the set of $3 \times 3$ skew symmetric matrices. We identify so(3) with $\mathbf{R}^{3}$ by identifying

$$
\mathrm{v}=(p, q, r) \in \mathbf{R}^{3} \quad \text { with } \quad \hat{\mathrm{v}}=\left[\begin{array}{ccc}
0 & -r & q \\
r & 0 & -p \\
-q & p & 0
\end{array}\right] \in \operatorname{so}(3) .
$$

The Lie bracket corresponds to the cross product in the sense that

$$
[\hat{\mathrm{v}}, \hat{w}]=(\mathrm{v} \times w)^{\hat{n}} .
$$

We denote elements of $s o(3)^{*}$ by $m$; these will also be identified with elements of $\mathbf{R}^{3}$. Elements $m \in s o(3)^{*}$ represent the body angular momentum of the rigid body and are related to the angular velocity $\omega$ by

$$
m_{i}=I_{i} \omega_{i}, \quad i=1,2,3
$$

where $I_{i}$ are the moments of inertia. As usual, the moment of inertia tensor has been diagonalized and we assume $I_{1} \geq I_{2} \geq I_{3}$. The standard Euler equations (Goldstein [1980, page 205]) written in terms of $m$ are 


$$
\left\{\begin{array}{l}
\dot{m}_{1}=\frac{I_{2}-I_{3}}{I_{2} I_{3}} m_{2} m_{3} \\
\dot{m}_{2}=\frac{I_{3}-I_{1}}{I_{1} I_{3}} m_{1} m_{3} \\
\dot{m}_{3}=\frac{I_{1}-I_{2}}{I_{1} I_{2}} m_{1} m_{2} .
\end{array}\right.
$$

Taking the Hamiltonian to be

$$
H(m)=\frac{1}{2} \sum_{j=1}^{3} \frac{m_{j}^{2}}{I_{j}}
$$

we see by a simple calculation that (3.4) are equivalent to

$$
\dot{F}=\{\{F, H\}\}
$$

where $\{\{\}$,$\} is the Lie-Poisson bracket. In the present case this bracket becomes$

$$
\{\{F, G\}\}(m)=-m \cdot(\nabla F \times \nabla G)
$$

for $F, G$ functions of $m$.

The fact that the equations (3.6) must preserve coadjoint orbits amounts in this case to the fact that

$$
\ell^{2}=m_{1}^{2}+m_{2}^{2}+m_{2}^{2}
$$

is an (obvious) constant of the motion for (3.4). In terms of coadjoint orbits, equations (3.4) are Hamiltonian on each sphere in $m$-space with Hamiltonian function (3.5). [The coadjoint orbits are spheres because the coadjoint action of $S O(3)$ on $m$ space is just by rotations. The coadjoint orbit symplectic structure is proportional to the area element as a simple calculation shows.]

The flow lines are given by intersecting the ellipsoids $H=$ constant with the spheres. For distinct moments of inertia the flow on the sphere has saddle points at $(0, \pm \ell, 0)$ and centers at $( \pm \ell, 0,0),(0,0, \pm \ell)$. The saddles are connected by four heteroclinic orbits, as indicated in Figure 1.

The orbits are, of course, explicitly known in terms of elliptic functions. The orbits of the most interest to us are the heteroclinic orbits which are given as follows. These four orbits lie in the invariant planes

$$
m_{3}= \pm \sqrt{\frac{a_{3}}{a_{1}}} m_{1}
$$

where

$$
a_{1}=\frac{I_{2}-I_{3}}{I_{2} I_{3}}>0, \quad a_{2}=\frac{I_{3}-I_{1}}{I_{1} I_{3}}<0 \quad \text { and } \quad a_{3}=\frac{I_{1}-I_{2}}{I_{1} I_{2}}>0 .
$$




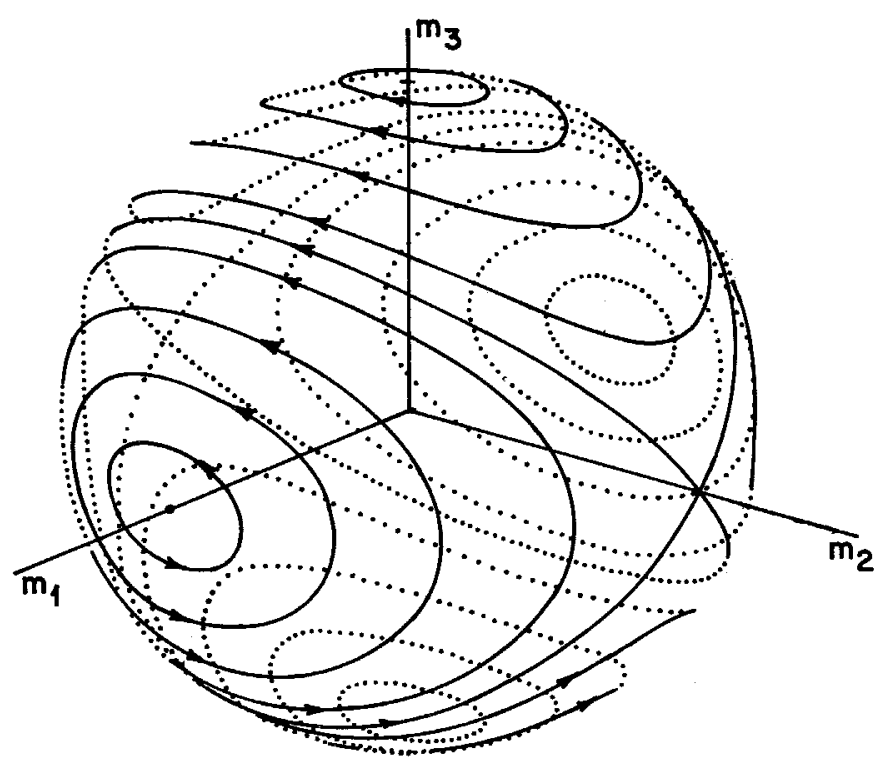

Figure 1. The spherical phase space of the rigid body for fixed total angular momentum $\ell=$ $\sqrt{m_{1}^{2}+m_{2}^{2}+m_{3}^{2}} ; I_{1}>I_{2}>I_{3}$.

3.1 Theorem. The heteroclinic orbits for the free rigid body are given by

$$
\begin{aligned}
& m_{1}^{+}(t)= \pm \ell \sqrt{\frac{a_{1}}{-a_{2}}} \operatorname{sech}\left(-\sqrt{a_{1} a_{3}} \ell t\right), \\
& m_{2}^{+}(t)= \pm \ell \tanh \left(-\sqrt{a_{1} a_{3}} \ell t\right), \\
& m_{3}^{+}(t)= \pm \ell \sqrt{\frac{a_{3}}{-a_{2}}} \operatorname{sech}\left(-\sqrt{a_{1} a_{3}} \ell t\right),
\end{aligned}
$$

for $m_{3}=+\left(\sqrt{a_{3} / a_{1}}\right) m_{1}$ and by

$$
m_{1}^{-}(t)=m_{1}^{+}(-t), m_{2}^{-}(t)=m_{2}^{+}(-t), m_{3}^{-}(t)=-m_{3}^{+}(-t)
$$

for $m_{3}=-\left(\sqrt{a_{3} / a_{1}}\right) m_{1}$.

This may be checked by direct computation or by consulting one of the classical texts.

(b) The heavy top: Euler angle description. We now recall the traditional Euler angle description of the heavy top and shall locate homoclinic orbits for the symmetric (Lagrange) top. The Euler angle description is more familiar but in some respects the Lie-Poisson description is simpler. For this reason we shall present both.

Given a rotation $A \in S O(3)$ we let the corresponding Euler angles be denoted 


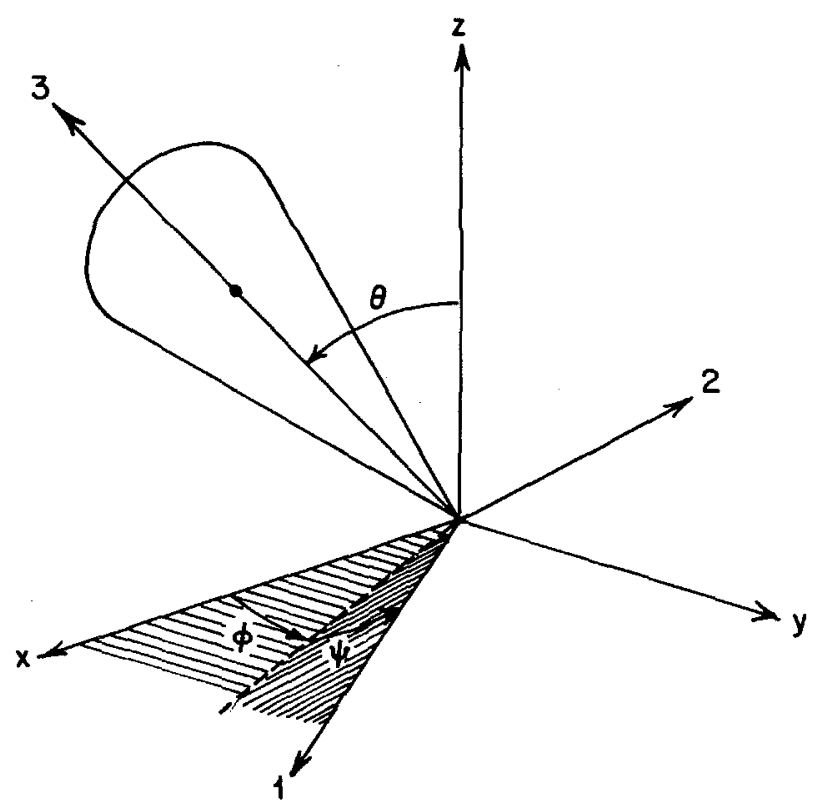

(a)

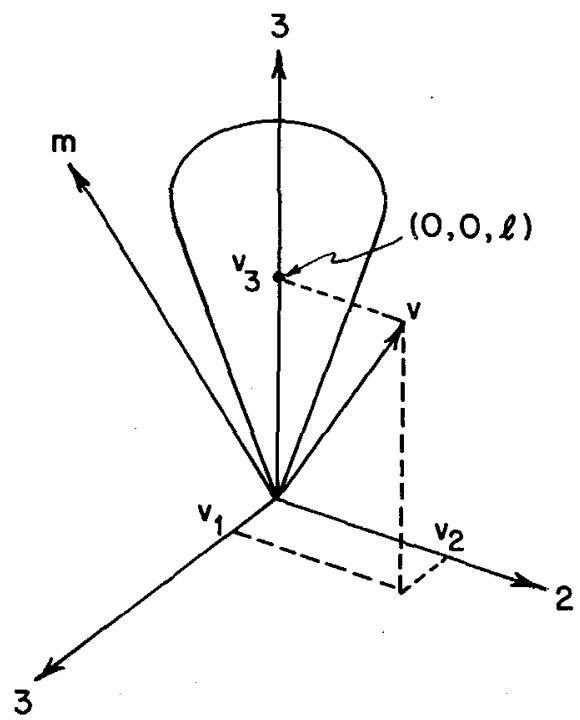

(b)

Figure 2. The heavy rigid body, illustrating space $(x, y, z)$ and body $(1,2,3)$ coordinates, and the Euler angles $(\phi, \psi, \theta)$. 
$(\phi, \psi, \theta)$ using the conventions of Goldstein [1980]; see Figure 2(a). The corresponding conjugate momenta are denoted $p_{\phi}, p_{\psi}, p_{\theta}$ so that $\left(\phi, \psi, \theta, p_{\phi}, p_{\psi}, p_{\theta}\right)$ coordinatize $T * S O(3)$.

We let $m$ denote the angular momentum in the body and let $\mathrm{v}=A^{-1} k$ where $k$ is the unit vector along the spatial $z$-axis. We assume the center of mass is at $(0,0, \ell)$ when $A$ is the identity. The vectors $(m, v)$ are expressed in the body coordinate system; see Figure 2(b).

The Hamiltonian is

$$
H(m, \mathrm{v})=\frac{1}{2} \sum_{j=1}^{3} \frac{m_{j}^{2}}{I_{j}}+M g \ell \mathrm{v}_{3}
$$

where $M$ is the total mass. When written in terms of Euler angles, this becomes

$$
\begin{aligned}
H=\frac{1}{2}\{ & \frac{\left[\left(p_{\phi}-p_{\psi} \cos \theta\right) \sin \psi+p_{\theta} \sin \theta \cos \psi\right]^{2}}{I_{1} \sin ^{2} \theta} \\
& \left.+\frac{\left[\left(p_{\theta}-P_{\psi} \cos \theta\right) \cos \psi-p_{\theta} \sin \theta \sin \psi\right]^{2}}{I_{2} \sin ^{2} \theta}+\frac{P_{\psi}^{2}}{I_{3}}\right\}+M g \ell \cos \theta .
\end{aligned}
$$

In Table 1 below we summarize the relationships among $m, v, \phi, \psi, \theta, p_{\phi}, p_{\psi}$, $p_{\theta}, \dot{\phi}, \dot{\psi}$ and $\dot{\theta}$ for convenient reference, but in this subsection we shall take (3.12), or equivalently the Lagrangian

$$
\begin{aligned}
L=\frac{I_{1}}{2}(\dot{\phi} \sin \theta \sin \psi+\dot{\theta} \cos \psi)^{2}+\frac{I_{2}}{2}(\dot{\phi} & \sin \theta \cos \psi-\dot{\theta} \sin \psi)^{2} \\
& +\frac{I_{3}}{2}(\dot{\phi} \cos \theta+\dot{\psi})^{2}-M g \ell \cos \theta
\end{aligned}
$$

as our starting point, and work exclusively in terms of Euler angles.

The Hamiltonian (3.12) is invariant under rotations about the $z$-axis; i.e. $\phi$ is a cyclic variable, so $p_{\phi}$ is a constant of the motion. In other terms, the momentum map for this $S^{1}$ action is $J\left(\phi, \psi, \theta, p_{\phi}, p_{\psi}, p_{\theta}\right)=p_{\phi}$. The reduced space $J^{-1}\left(p_{\phi}\right) / S^{1}$ is parametrized by $\left(\psi, \theta, p_{\psi}, p_{\theta}\right)$. In fact this reduced space is identifiable with $T^{*} S^{2}$, the cotangent bundle of the two sphere on which $\left(\psi, \theta, p_{\psi}, p_{\theta}\right)$ are canonical coordinates. As we shall see below, $(\psi, \theta)$ is a system of spherical coordinates for this sphere. The equations of motion for $\psi, \theta$ are thus just Hamilton's equations for (3.12) with $p_{\phi}$ held constant.

Remarks 1. The $S^{1}$ reduction here is in accord with the general fact that reducing $T^{*} Q$ by $S^{1}$ gives $T^{*}\left(Q / S^{1}\right)$; here $Q=S O(3)$ and $S O(3) / S^{1}=S^{2}$.

2. The two sphere obtained here is not to be confused with the sphere for the free rigid body shown in Figure 1.

For the symmetric top (i.e., for $I_{1}=I_{2}$ ), $\psi$ is also a cyclic variable and $p_{\psi}$ is constant as well. In fact these two $S^{1}$ symmetries commute, so we have a two- 
torus $\left(T^{2}\right)$ symmetry, which makes the system completely integrable. The reduced system has one degree of freedom, namely $\left(\theta, p_{\theta}\right)$. The reduced space is no longer a manifold, but has a boundary at $\theta=0, \pi$; i.e. it is $T^{*}[0, \pi]$. These singularities in the reduced space correspond to the fact that the level set $p_{\psi}=$ constant is singular at $\theta=0, \pi$. [This is a special case of a general fact about singularities in level sets of momentum maps; see Arms, Marsden and Moncrief [1981].]

With $I_{1}=I_{2}$, (3.12) becomes

$$
\begin{aligned}
H & =\frac{1}{2 I_{1} \sin ^{2} \theta}\left\{\left(p_{\phi}-p_{\psi} \cos \theta\right)^{2}+p_{\theta}^{2} \sin ^{2} \theta\right\}+\frac{p_{\psi}^{2}}{I_{3}}+M g \ell \cos \theta \\
& =\frac{p_{\theta}^{2}}{2 I_{1}}+\left(M g \ell \cos \theta+\frac{\left(p_{\phi}-p_{\psi} \cos \theta\right)^{2}}{2 I_{1} \sin ^{2} \theta}+\frac{p_{\psi}^{2}}{I_{3}}\right)
\end{aligned}
$$

which shows the amended potential explicitly.

Remark. In these coordinates, the abstract formula (2.9) for the one forms $\alpha_{\mu}$ associated with the $\phi, \psi$-reductions gives the closed one forms $p_{\phi} d p_{\phi}$ and $p_{\psi} d p_{\psi}$ respectively. Interestingly, this means that the corresponding "magnetic term" is singular, being supported at the two singular points $\psi=0$ and $\pi$.

3.2 Theorem. If $0<p<2 \sqrt{M g \ell I_{1}}$ and if $p_{\phi}=p_{\psi}=I_{1} b$, the reduced Lagrange top system in $\left(\theta, p_{\theta}\right)$ space has a hyperbolic saddle point at $\theta=0, p_{\theta}=$ 0 and a homoclinic orbit connecting it to itself given by

$$
\cos \theta=1-\gamma \operatorname{sech}^{2}\left(\frac{\sqrt{\beta \gamma} t}{2}\right),
$$

where $\beta=2 \mathrm{Mgl} / \mathrm{I}_{1}$ and $\gamma=2-b^{2} / \beta$.

Remarks 1. A top with $0<p_{\psi}<2 \sqrt{M g \ell I_{1}}$ for which the vertically spinning state is unstable (a saddle) is called a slow top.

2. For $p_{\phi}=p_{\psi}$ note that the potential in (3.14) does not have a singularity at $\theta=0, \pi$, so these endpoints do not cause difficulties.

3. Note that $0<\gamma<2$.

The theorem is most easily checked by using the energy equation (3.14); the homoclinic orbit has energy $H=M g \ell+P_{\psi}^{2} / 2 I_{3}$; cf. Goldstein [1980, pages 215, 216] and Figure 3.

(c) The heavy top: Lie-Poisson description. The abstract theory in the preceding section guarantees that the reduced space $T^{*} S^{2}$ for the heavy top is symplectically diffeomorphic to a coadjoint orbit in the semi-direct product $S O(3) \times$ $\mathbf{R}^{3}$; i.e. in the Euclidean group $E_{3}$. The Lie algebra is denoted $e_{3}$.

The mapping that gives this diffeomorphism is just the map

$$
\Lambda:\left(\phi, \psi, \theta, p_{\phi}, p_{\psi}, p_{\theta}\right) \mapsto(m, \mathrm{v})
$$




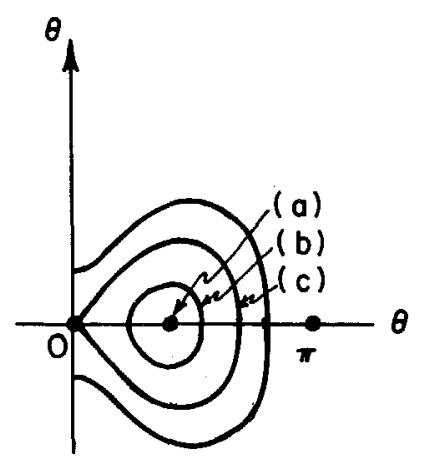

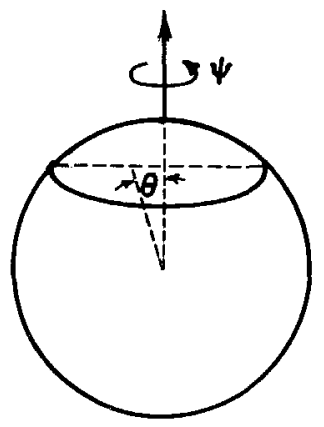

(a)

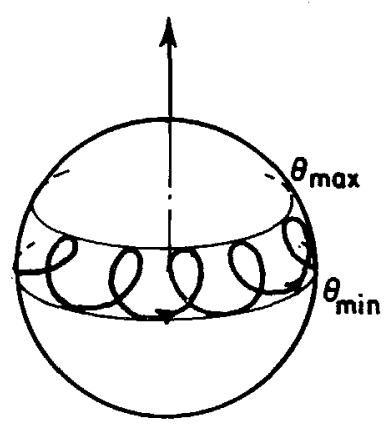

(b)

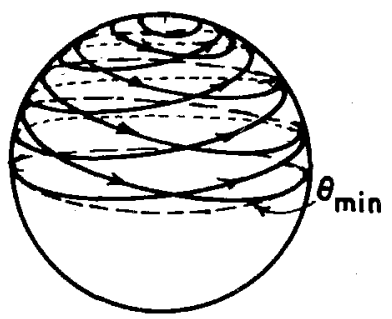

(c)

FiguRE 3. Motions of the top a) steady precession $(\theta=$ constant $)$ b) precession and nutation $\left.\left(\theta_{\min } \leq \theta \leq \theta_{\max }\right) c\right)$ homoclinic orbit $\left(\theta_{\min } \leq \theta<0\right)$.

where $m$ is the angular momentum in the body and $\mathrm{v}$ is the orientation of gravity as viewed from the body. Table 1 below summarizes the explicit formulas relating these quantities. Table 2 summarizes the relationships between the "Euler angle" spaces and the coadjoint spaces.

The Lie-Poisson bracket for functions of $(m, v)$ is given by (2.2) and (2.15), which in this case becomes

$$
\{\{F, G\}\}(m, \mathrm{v})=-m \cdot\left(\nabla_{m} F \times \nabla_{m} G\right)-\mathrm{v} \cdot\left(\nabla_{m} F \times \nabla_{\mathrm{v}} G+\nabla_{\mathrm{v}} F \times \nabla_{m} G\right) .
$$

The assertions of the general theory can be checked by hand in this case. First of all, it is easily seen that the Lie-Poisson bracket equation $\dot{F}=\{\{F, H\}\}$ with $H$ given by (3.11) and the bracket by (3.17) yield the equations

$$
\left\{\begin{array}{l}
\dot{m}_{1}=a_{1} m_{2} m_{3}-M g \ell \mathrm{v}_{2} \\
\dot{m}_{2}=a_{2} m_{1} m_{3}+M g \ell \mathrm{v}_{1} \\
\dot{m}_{3}=a_{3} m_{1} m_{2}
\end{array}\right.
$$




$$
\left\{\begin{array}{l}
\dot{\mathrm{v}}_{1}=\frac{m_{3} \mathrm{v}_{2}}{I_{3}}-\frac{m_{2} \mathrm{v}_{3}}{I_{2}} \\
\dot{\mathrm{v}}_{2}=\frac{m_{1} \mathrm{v}_{3}}{I_{1}}-\frac{m_{3} \mathrm{v}_{1}}{I_{3}} \\
\dot{\mathrm{v}}_{3}=\frac{m_{2} \mathrm{v}_{1}}{I_{2}}-\frac{m_{1} \mathrm{v}_{2}}{I_{1}}
\end{array}\right.
$$

A slightly tedious, though straightforward computation shows that these are equivalent to the Hamiltonian equations for (3.12). In fact, if $F, G$ are functions of $(m, \mathrm{v})$ and $(m, \mathrm{v})$ are then written out in terms of the Euler variables then an explicit computation shows that

$$
\{\{F, G\}\}(m, \mathrm{v})=\{F, G\}_{\theta, \psi}
$$

where $\{,\}_{\theta, \psi}$ denotes the bracket computed for the $\theta, \psi$ variables (i.e. holding $p_{\phi}$ constant). This is the content of Theorem 2.1 for this special case.

TABLE 1-Formulas Relating Euler Variables and Lie-Poisson Variables for the Heavy Top

$m_{1}=\left[\left(p_{\phi}-p_{\psi} \cos \theta\right) \sin \psi+p_{\theta} \sin \theta \cos \psi\right] / \sin \theta=I_{1}(\dot{\phi} \sin \theta \sin \psi+\dot{\theta} \cos \psi)$
$m_{2}=\left[\left(p_{\phi}-p_{\psi} \cos \theta\right) \cos \psi-p_{\theta} \sin \theta \sin \psi\right] / \sin \theta=I_{2}(\dot{\phi} \sin \theta \cos \psi-\dot{\theta} \sin \psi)$
$m_{3}=p_{\psi}=I_{3}(\dot{\phi} \cos \theta+\dot{\psi})$
$\mathrm{v}_{1}=\sin \theta \sin \psi$
$\mathrm{v}_{2}=\sin \theta \cos \psi$
$\mathrm{v}_{3}=\cos \theta$
$p_{\phi}=m \cdot \mathrm{v}=I_{1}(\dot{\phi} \sin \theta \sin \psi+\dot{\theta} \cos \psi) \sin \theta \sin \psi$
$p_{\phi}=+I_{2}(\dot{\phi} \sin \theta \cos \psi-\dot{\theta} \sin \psi) \sin \theta \cos \psi+I_{3}(\dot{\phi} \sin \theta+\dot{\psi}) \cos \theta$
$p_{\psi}=m_{3}=I_{3}(\dot{\phi} \cos \theta+\dot{\psi})$
$p_{\theta}=\left(\mathrm{v}_{2} m_{1}-\mathrm{v}_{1} m_{2}\right) / \sqrt{1-\mathrm{v}_{3}^{2}}=I_{1}(\dot{\phi} \sin \theta \sin \psi+\dot{\theta} \cos \psi) \cos \psi$
$p_{\theta}=-I_{2}(\dot{\phi} \sin \theta \cos \psi-\dot{\theta} \sin \psi) \sin \psi$
$\dot{\phi}=\frac{m_{1} \mathrm{v}_{1}}{I_{1}\left(1-\mathrm{v}_{3}^{2}\right)}+\frac{m_{2} \mathrm{v}_{2}}{I_{2}\left(1-\mathrm{v}_{3}^{2}\right)}$
$\dot{\psi}=\frac{m_{3}}{I_{3}}-\frac{m_{3} m_{1} \mathrm{v}_{1}}{I_{1}\left(1-\mathrm{v}_{3}^{2}\right)}-\frac{m_{3} m_{2} \mathrm{v}_{2}}{I_{2}\left(1-\mathrm{v}_{3}^{2}\right)}$
$\dot{\theta}=\frac{m_{1} \mathrm{v}_{2}}{I_{1} \sqrt{1-\mathrm{v}_{3}^{2}}}-\frac{m_{2} \mathrm{v}_{1}}{I_{2} \sqrt{1-\mathrm{v}_{3}^{2}}}$


TABLE 2-The Relationship Among the Spaces and Variables

\begin{tabular}{|c|c||l|c|}
\hline \multicolumn{2}{|c||}{ Euler Angles } & \multicolumn{2}{c|}{ Lie-Poisson } \\
\hline Variables & \multicolumn{1}{|c|}{ Space } & Space & Variables \\
\hline$\left(\phi, \psi, \theta, p_{\phi}, p_{\psi}, p_{\theta}\right)$ & $T^{*} S O(3)$ & $e_{3}^{*}$ & $(m, \mathrm{v})$ \\
& $\begin{array}{l}\text { reduce by } \\
S^{1}: \phi \text { cyclic }\end{array}$ & $\downarrow$ & \\
$\left(\psi, \theta, p_{\psi}, p_{\theta}\right)$ & $T^{*} S^{2}$ \\
& $\begin{array}{l}\text { If } I_{1}=I_{2}, \\
\text { Lagrange } \\
\text { reduction }\end{array}$ & $\mid \begin{array}{l}\text { restriction } \\
\text { coadjoint orbit }\end{array}$ & $\|\mathrm{v}\|=1, m \cdot \mathrm{v}=p_{\phi}$ \\
$\left(\theta, p_{\theta}\right)$ & $T^{*}[0, \pi]$ & & \\
\hline
\end{tabular}

The equations (3.18) and (3.19) have $\|\mathrm{v}\|$ and $m \cdot \mathrm{v}$ as constants of the motion. This just reflects (a) the conservation law $p_{\phi}=$ constant and (b) the preservation of the coadjoint orbit by the Lie-Poisson equations. The conditions $\|\mathrm{v}\|=1$ and $m \cdot \mathrm{v}=p_{\phi}=$ constant also give the explicit identification of the coadjoint orbit with $T^{*} S^{2}$. Indeed, $\|\mathrm{v}\|=1$ describes the unit sphere $S^{2}$ and $m \cdot \mathrm{v}=p_{\phi}$ specifies $m$ as a linear functional on the unit normal to $S^{2}$, leaving $m$ restricted to $T_{\mathrm{v}} S^{2}$ free. Thus $m$ determines, by restriction, an element of $T_{v}^{*} S^{2}$. Finally observe that the equations for $\mathrm{v}$ in Table 1 show that $\theta, \psi$ are spherical-type coordinates on $S^{2}$ ( $\psi$ has been rotated by $\pi / 2$ from standard conventions on spherical coordinates).

Finally we discuss the Lagrange top in the Lie-Poisson picture. For $I_{1}=I_{2}$ the invariance is rotation about the 3-axis. This $S^{1}$ action corresponds to the $S^{1}$ action of rotation through $\psi$ in the Euler angle picture, as is easily seen. Also, the momentum map can be directly checked to be just $m_{3}$.

The following is a general property of reduction, but it may also be checked explicitly in this case.

3.3 Lemma. If $F$ and $G$ are functions of $(m, v)$ which are rotationally invariant, then

$$
\{\{F, G\}\}=\{\{F, G\}\}_{m_{3}-\text { const }}
$$

i.e. the full Lie-Poisson bracket is the same as if it were computed holding $m_{3}$ constant.

With $\|\mathrm{v}\|=1, m \cdot \mathrm{v}=p_{\phi}$, any rotationally invariant function of $(m, \mathrm{v})$ can in fact be expressed as a function of $\mathrm{v}_{2} m_{1}-\mathrm{v}_{1} m_{2}$ and $\mathrm{v}_{3}$. These variables are a convenient representation of the coadjoint space reduced by the $S^{1}$ action. Brackets of functions of these variables may be computed by Lemma 3.3. Again, one can check by hand that for such functions

$$
\{\{F, G\}\}=\{F, G\}_{\theta}
$$


where

$$
\{F, G\}_{\theta}=\frac{\partial F}{\partial \theta} \frac{\partial G}{\partial p_{\theta}}-\frac{\partial G}{\partial \theta} \frac{\partial F}{\partial p_{\theta}} ;
$$

i.e. the canonical bracket holding $p_{\phi}, p_{\psi}$ constant.

The homoclinic orbit now is described very simply as follows:

3.4 Theorem. For $0<m_{3}<2 \sqrt{M g \ell I_{1}}$ and $m \cdot v=m_{3}$, the reduced system for the heavy top has a hyperbolic saddle point at

$$
\mathrm{v}_{3}=1, \quad \mathrm{v}_{2} m_{1}-\mathrm{v}_{1} m_{2}=0
$$

and a homoclinic orbit connecting it to itself given by

$$
\left\{\begin{aligned}
\mathrm{v}_{3} & =1-\gamma \operatorname{sech}^{2}\left(\frac{\sqrt{\beta \gamma} t}{2}\right) \text { and } \\
\mathrm{v}_{2} m_{1}-\mathrm{v}_{1} m_{2} & =-I_{1} \dot{\mathrm{v}}_{3}\left[=-I_{1} \dot{\theta} \sin \theta\right]
\end{aligned}\right.
$$

where $\beta, \gamma$ are as in Theorem 3.2 with $p_{\psi}=m_{3}$.

This can be checked directly (using the energy equation and the evolution equations). Of course it corresponds to (3.15).

\section{§4. Transversal Intersections of Invariant Manifolds for $K K S$ Variables Coupled to Action Angle Variables}

We now develop an extension of the Melnikov-Arnold theory (Melnikov [1963], Arnold [1964], Holmes [1980]) which applies to systems described by a set of $m$ Lie-Poisson variables $\mu=\left(\mu_{1}, \ldots, \mu_{m}\right) \in g^{*}$ and a set of $n$ action angles $\left(\theta_{1}, I_{1}\right)$, $\ldots,\left(\theta_{n}, I_{n}\right)$. This theory will then be applied to the free rigid body with attachments in the next section. For the heavy top, a more sophisticated version is needed for systems whose variables do not decompose so cleanly. This is the subject of Section 6.

The action angle variables can be those associated with motion near an elliptic fixed point in a one degree of freedom system. For example, our methods apply to the system obtained by coupling two rigid bodies if we examine the motion near a homoclinic orbit in one (such as an orbit connecting $(0, \ell, 0)$ to $(0,-\ell, 0)$ in Figure 1) and a small periodic orbit in the other (such as an orbit near $(\ell, 0,0)$ in Figure 1). The coupled system then will have horseshoes under the conditions of Theorem 4.3 below. (We believe that this applies, in particular, to the five mode truncation of the Euler equations for an ideal fluid on a two-torus, which consists of two sets of overlapping and coupled rigid body equations; note that for $g=s o(3)$ and $n=1$, we have five variables in the set $(\mu, \theta, I)$.) For simplicity, however, we have chosen a rigid body with attachments to work out in detail since the action angle variables are more explicit and it is easy to add on additional ones. 
We assume that our Hamiltonian takes the form

$$
\begin{aligned}
H^{\mathrm{\varepsilon}}(\mu, \theta, I) & =F(\mu)+\sum_{j=1}^{n} G_{j}\left(I_{j}\right)+\varepsilon H^{1}(\mu, \theta, I) \\
& =H^{0}\left(\mu, I_{1}, \ldots, I_{n}\right)+\varepsilon H^{1}(\mu, \theta, I)+O\left(\varepsilon^{2}\right)
\end{aligned}
$$

where $\mu=\left(\mu_{1}, \ldots, \mu_{m}\right) \in \mathfrak{g}^{*}$, the dual space of a Lie algebra $\mathfrak{g}$ and $\theta=$ $\left(\theta_{1}, \ldots, \theta_{n}\right), I=\left(I_{1}, \ldots, I_{n}\right)$ with $\theta_{i}$ a $2 \pi$-periodic variable. We assume that the system associated with $F$ has a homoclinic (or heteroclinic) orbit $\bar{\mu}(t) \in \mathrm{g}^{*}$. The oscillator frequencies

$$
\Omega_{j}\left(I_{j}\right)=\frac{\partial G_{j}}{\partial I_{j}}
$$

are assumed to be positive.

As in Holmes and Marsden [1982a], [1982b] we can solve the equation

$$
H^{\varepsilon}(\mu, \theta, I)=h
$$

for $I_{n}$ in the form

$$
\begin{aligned}
I_{n} & =L^{\varepsilon}\left(\mu, \theta_{1}, \ldots, \theta_{n}, I_{1}, \ldots, I_{n-1}, h\right) \\
& =L^{0}\left(\mu, I_{1}, \ldots, I_{n-1}, h\right)+\varepsilon L^{1}\left(\mu, \theta_{1}, \ldots, \theta_{n}, I_{1}, \ldots, I_{n-1}, h\right)+O\left(\varepsilon^{2}\right)
\end{aligned}
$$

where

$$
L^{0}\left(\mu, I_{1}, \ldots, I_{n-1}, h\right)=G_{n}^{-1}\left(h-F(\mu)-\sum_{j=1}^{n-1} G_{j}\left(I_{j}\right)\right)
$$

and

$$
\begin{aligned}
L^{1}\left(\mu, \theta_{1}, \ldots, \theta_{n}, I_{1}, \ldots, I_{n-1}, h\right) & \\
& =-\frac{H^{1}\left(\mu, \theta_{1}, \ldots, \theta_{n}, I_{1}, \ldots, I_{n-1}, L^{0}\left(\mu, I_{1}, \ldots, I_{n-1}, h\right)\right)}{\Omega_{n}\left(L^{0}\left(\mu, I_{1}, \ldots, I_{n-1}, h\right)\right)} .
\end{aligned}
$$

In addition, we eliminate $t$ in favor of the new 'time' $\theta_{n}$ and write ' for $d / d \theta_{n}$.

4.1 Proposition. Hamilton's equation for (4.1), namely

$$
\left\{\begin{array}{l}
\dot{\mu}_{i}=\left\{\left\{\mu_{i}, H^{\varepsilon}\right\}\right\}, \quad i=1, \ldots, k \\
\dot{\theta}_{j}=\frac{\partial H^{\varepsilon}}{\partial I_{j}}, \quad \dot{I}_{j}=-\frac{\partial H^{\varepsilon}}{\partial \theta_{j}}, \quad j=1, \ldots, n,
\end{array}\right.
$$

(where $\{\{\}$,$\} denotes the Lie-Poisson bracket in the \mu$-variables) become

$$
\begin{aligned}
& \mu_{i}^{\prime}=-\left\{\left\{\mu_{i}, L^{\varepsilon}\right\}\right\}, \quad i=1, \ldots, k \\
& \theta_{j}^{\prime}=-\frac{\partial L^{\varepsilon}}{\partial I_{j}}, \quad I_{j}^{\prime}=\frac{\partial L^{\varepsilon}}{\partial \theta_{j}}, \quad j=1, \ldots, n-1 .
\end{aligned}
$$


Proof. Implicit differentiation of (4.3) gives

$$
\frac{\delta H^{\varepsilon}}{\delta \mu}+\frac{\partial H^{\mathrm{\varepsilon}}}{\partial I_{n}} \frac{\delta L^{\mathrm{\varepsilon}}}{\delta \mu}=0
$$

It follows that for a function $K(\mu)$,

$$
\left\{\left\{K, H^{\varepsilon}\right\}\right\}(\mu)=-\left\langle\mu,\left[\frac{\delta K}{\delta \mu}, \frac{\delta H^{\varepsilon}}{\delta \mu}\right]\right\rangle=\left\langle\mu,\left[\frac{\delta K}{\delta \mu}, \frac{\partial H^{\varepsilon}}{\partial I_{n}} \frac{\delta L^{\varepsilon}}{\delta \mu}\right]\right\rangle
$$

and so $\left\{\left\{K, H^{\varepsilon}\right\}\right\} /\left(\partial H^{\varepsilon} / \partial I_{n}\right)=-\left\{\left\{K, L^{\varepsilon}\right\}\right\}$. However then,

$$
K^{\prime}=\frac{\dot{K}}{\dot{\theta}_{n}}=\frac{\dot{K}}{\partial H^{\varepsilon} / \partial I_{n}}=-\left\{\left\{K, L^{\varepsilon}\right\}\right\} .
$$

The second set of equations in (4.8) follows in the same way.

Using $L^{\varepsilon}=L^{0}+\varepsilon L^{1}+O\left(\varepsilon^{2}\right)$ we see that (4.8) has the form of a periodically perturbed system. Next we relate the reduced and nonreduced brackets.

4.2 Proposition. We have

$$
\left\{\left\{L^{0}, L^{1}\right\}\right\}=\frac{1}{\left(\Omega_{n}\right)^{2}}\left\{\left\{F, H^{1}\right\}\right\} .
$$

Proof. From (4.5),

$$
\frac{\delta L^{0}}{\delta \mu}=-\frac{1}{\Omega_{n}} \frac{\delta F}{\delta \mu}
$$

and

$$
\frac{\delta L^{1}}{\delta \mu}=-\frac{1}{\Omega_{n}}\left(\frac{\delta H^{1}}{\delta \mu}+\frac{\partial H^{1}}{\partial I_{n}} \frac{\delta L^{0}}{\delta \mu}\right)+\frac{1}{\left(\Omega_{n}\right)^{2}} H^{1} \frac{\partial \Omega_{n}}{\partial I_{n}} \frac{\delta L^{0}}{\delta \mu} .
$$

However, since $\left[\delta L^{0} / \delta \mu, \delta L^{0} / \delta \mu\right]=0$, we get

$$
\begin{aligned}
\left\{\left\{L^{0}, L^{1}\right\}\right\} & =-\left\langle\mu,\left[\frac{\delta L^{0}}{\delta \mu}, \frac{\delta L^{1}}{\delta \mu}\right]\right\rangle \\
& =-\left\langle\mu, \frac{1}{\Omega_{n}^{2}}\left[\frac{\delta F}{\delta \mu}, \frac{\delta H^{1}}{\delta \mu}\right]\right\rangle \\
& =\frac{1}{\Omega_{n}^{2}}\left\{\left\{F, H^{1}\right\}\right\} .
\end{aligned}
$$

Let us now give a special case of the general result, suitable for two degree of freedom systems.

4.3 Theorem. Suppose $\bar{\mu}(t)$ is a homoclinic (or heteroclinic) orbit for $F$, which lies on a coadjoint orbit in $\mathfrak{g}^{*}$ of dimension 2. Furthermore, suppose $n=1$. 
Let $\bar{h}=F(\bar{\mu})$ be the energy of the homoclinic orbit and let $h>\bar{h}$ and $\ell^{0}=$ $G^{-1}(h-\bar{h})$ be constants. Let $\left\{\left\{F, H^{1}\right\}\right\}\left(t, \theta^{0}\right)$ denote the Lie-Poisson bracket of $F(\mu)$ and $H^{1}\left(\mu, \Omega\left(\ell^{0}\right) t+\theta^{0}, \ell^{0}\right)$ evaluated at $\bar{\mu}(t)$. Let

$$
M\left(\theta^{0}\right)=\frac{1}{\Omega_{n}\left(\ell^{0}\right)} \int_{-\infty}^{\infty}\left\{\left\{F, H^{1}\right\}\right\}\left(t, \theta^{0}\right) d t
$$

and assume $M\left(\theta^{0}\right)$ has simple zeros. Then for $\varepsilon>0$ sufficiently small, the Hamiltonian system (4.1) contains transverse homoclinic orbits and hence Smale horseshoes on the energy surface $H^{\varepsilon}=h$.

Proof. By reduction, it suffices to check that the Melnikov function for the reduced, forced system on the coadjoint orbit containing $\bar{\mu}$ has simple zeros. This involves only a generalization of the one degree of freedom Melnikov theory for forced oscillations to two-dimensional symplectic manifolds. The standard proof (see Holmes [1980] and Greenspan and Holmes [1981]) carries over directly. The Melnikov function for the reduced system is

$$
M\left(\theta^{0}\right)=\int_{-\infty}^{\infty}\left\{\left\{L^{0}, L^{1}\right\}\right\} d \theta
$$

since the Lie-Poisson bracket coincides with the Poisson bracket on coadjoint orbits. Using (4.9) and $d \theta / d t=\Omega$,

$$
M\left(\theta^{0}\right)=\int_{-\infty}^{\infty} \frac{1}{\Omega}\left\{\left\{F, H^{1}\right\}\right\} d t .
$$

Finally, note that $\Omega=\Omega\left(\ell^{0}\right)$ is constant on the homoclinic orbit, so the theorem follows.

To deal with the situation in which $n \geq 2$, we introduce the following conditions on the Hamiltonian (4.1).

(H1) $F$ contains a homo- (or hetero-) clinic orbit $\bar{\mu} \in \mathrm{g}^{*}$ with energy $\bar{h}$. The coadjoint orbit containing $\tilde{\mu}$ is assumed to be two-dimensional. The saddle points for $\bar{\mu}$ are denoted $\mu_{ \pm}$(they could be coincident).

(H2) $\Omega_{j}\left(I_{j}\right)=G_{j}^{\prime}\left(I_{j}\right)>0, j=1, \ldots, n$.

To explain the remaining conditions some discussion is needed.

For $\varepsilon=0$, note that the Hamiltonian system for $L^{0}$ has two $(n-1)$-parameter families of invariant $(n-1)$-dimensional tori $T_{ \pm}\left(h_{1}, \ldots, h_{n-1}\right)$ given by

$$
\left\{\begin{array}{l}
\mu=\mu_{ \pm} \\
G_{j}\left(I_{j}\right)=h_{j}=\text { constant } \quad\left(\text { i.e. } I_{j}=\ell_{j}=G_{j}^{-1}\left(h_{j}\right)\right) \\
\theta_{j}=\Omega_{j}\left(\ell_{j}\right) \theta_{n}+\theta_{j}(0) \quad(\bmod 2 \pi), \quad j=1, \ldots, n-1 .
\end{array}\right.
$$

(Correspondingly, the system for $H^{0}$ has two $n$-parameter families of invariant tori $T_{ \pm}\left(h_{1}, \ldots, h_{n}\right)$.) Henceforth we write the (phase) constants of integration $\theta_{j}(0)$ as $\theta_{j}^{0}, j=1, \ldots, n-1, n$. 
The tori $T_{ \pm}\left(h_{1}, \ldots, h_{n-1}\right)$ are connected by the $n$-dimensional homoclinic manifold defined by

$$
\left\{\begin{array}{l}
\mu=\bar{\mu}\left(\theta_{n}-\theta_{n}^{0}\right) \\
G_{j}\left(I_{j}\right)=h_{j}, \\
\theta_{j}=\Omega_{j}\left(I_{j}\right) \theta_{n}+\theta_{j}^{0}, \quad j=1, \ldots, n-1,
\end{array}\right.
$$

where the phase constant $\theta_{n}^{0}$ associated with the 'reduced' degree of freedom appears explicitly. This manifold consists of the coincident stable and unstable manifolds of the tori $T_{ \pm}\left(h_{1}, \ldots, h_{n-1}\right)$; i.e.

$$
W^{s}\left(T_{ \pm}\left(h_{1}, \ldots, h_{n-1}\right)\right)=W^{u}\left(T_{\mp}\left(h_{1}, \ldots, h_{n-1}\right)\right)
$$

given by (4.13).

For $\varepsilon \neq 0$ the system (4.7) possesses a Poincare map $P_{\varepsilon}$ from (a piece of) $\left(\mu, \theta_{1}, \ldots, \theta_{n-1}, I_{1}, \ldots, I_{n-1}\right)$ space to itself where $\theta_{n}$ goes through an increment of $2 \pi$, starting at some fixed value $\theta_{n}^{0}$, (which will be suppressed in the notation). Below, when we refer to transverse intersection of stable and unstable manifolds, we mean so for this Poincaré map.

(H3) Assume that the constants $G_{j}\left(I_{j}\right)=h_{j}, j=1, \ldots, n$ are chosen so that the unperturbed frequencies $\Omega_{1}\left(I_{1}\right), \ldots, \Omega_{1}\left(I_{n}\right)$ satisfy the nondegeneracy conditions (i.e. $\left.\Omega_{j}^{\prime}\left(I_{j}\right) \neq 0, j=1, \ldots, n-1\right)$ and the nonresonance conditions of the KAM theorem. (cf. Arnold [1978, Appendix 8]).

This condition ensures that the tori $T_{ \pm}\left(h_{1}, \ldots, h_{n-1}\right)$ perturb to invariant tori $T_{\varepsilon \pm}\left(h_{1}, \ldots, h_{n-1}\right)$ for $P_{\varepsilon}$ with $\varepsilon$ sufficiently small.

Let $h>\bar{h}, h=\bar{h}+\sum_{j=1}^{n} h_{j}$ where $h_{j}>0$ and the unperturbed homoclinic manifold be filled with an $n$-parameter family of orbits given by

$$
\left(\mu, \theta_{1}, \ldots, \theta_{n}, I_{1}, \ldots, I_{n}\right)=\left(\bar{\mu}(t), \Omega_{1}\left(I_{1}\right) t+\theta_{1}^{0}, \ldots, \Omega_{n}\left(I_{n}\right) t+\theta_{n}^{0}, I_{1}, \ldots, I_{n}\right) .
$$

Pick one such orbit and let $\left\{\left\{F, H^{1}\right\}\right\}$ denote the Lie-Poisson bracket of $F(\mu)$ and $H^{1}\left(\mu, \theta_{1}, \ldots, \theta_{n}, I_{1}, \ldots, I_{n}\right)$ evaluated on this orbit. Similarly, let $\left\{I_{k}, H^{1}\right\}=$ $-\partial H^{1} / \partial \theta_{k}, k \equiv 1, \ldots, n-1$ be evaluated on this orbit. Define the Melnikov Vector, $M\left(\theta^{0}\right)=\left(M_{1}, \ldots, M_{n-1}, M_{n}\right)$, by

$$
\begin{aligned}
& M_{k}\left(\theta_{1}^{0}, \ldots, \theta_{n}^{0}, h, h_{1}, h_{2}, \ldots, h_{n-1}\right)=\int_{-\infty}^{\infty}\left\{I_{k}, H^{1}\right\} d t, \quad k=1, \ldots, n-1 \\
& M_{n}\left(\theta_{1}^{0}, \ldots, \theta_{n}^{0}, h, h_{1}, h_{2}, \ldots, h_{n-1}\right)=\frac{1}{\Omega_{n}} \int_{-\infty}^{\infty}\left\{\left\{F, H^{1}\right\}\right\} d t .
\end{aligned}
$$

(We note that $h_{n}=h-\bar{h}-\sum_{j=1}^{n-1} h_{j} ; I_{n}$ and $h_{n}$ do not explicitly enter the calculations, since $I_{n}$ is eliminated by the reduction process; we also note that these integrals need not be absolutely convergent, but we do require conditional convergence for $M_{k}$, with appropriately chosen limits of integration (to suppress spurious oscillatory terms corresponding to motion on the torus.) 
(H4) Assume that the multiply $2 \pi$-periodic Melnikov vector $M: \mathbf{R}^{n} \rightarrow \mathbf{R}^{n}$ (which is independent of $\varepsilon$ ) has at least one transversal zero; i.e. there is a point $\left(\theta_{1}^{0}, \ldots, \theta_{n}^{0}\right)$ for which

$$
M\left(\theta_{1}^{0}, \ldots, \theta_{n}^{0}\right)=0
$$

but

$$
\operatorname{det}\left[D M\left(\theta_{1}^{0}, \ldots, \theta_{n}^{0}\right)\right] \neq 0,
$$

where $D M$ is the $n \times n$ matrix of partial derivatives of $M_{1}, \ldots, M_{n}$ with respect to $\theta_{1}^{0}, \ldots, \theta_{n}^{0}$, the initial phases of the orbit.

Here is the result for $n \geq 2$.

4.4 Theorem. If conditions ( $H 1)-(H 4)$ hold for the system (4.1), then, for $\varepsilon$ sufficiently small, the perturbed stable and unstable manifolds $W^{s}\left(T_{\varepsilon, \pm}\right)$, and $W^{u}\left(T_{\varepsilon+}\right)$ of the perturbed tori $T_{\varepsilon, \pm}$ intersect transversely.

The theorem follows from the arguments of Holmes and Marsden [1982b] in the present context. We also refer the reader to that paper for a discussion of how this yields Arnold diffusion and for related references.

There is a similar result when the coadjoint orbit is higher dimensional i.e. of dimension $2 M, M>1$, but the system for $F$ on $\mathfrak{g}^{*}$ is completely integrable, say with integrals

$$
F=F_{1}, F_{2}, \ldots, F_{M},
$$

where $F_{2}, \ldots, F_{M}$ are associated with action angle variables. Now a result similar to 4.4 holds if the Melnikov vector is enlarged by replacing the function

$$
\frac{1}{\Omega_{n}} \int_{-\infty}^{\infty}\left\{\left\{F, H^{1}\right\}\right\} d t
$$

by the vector

$$
\frac{1}{\Omega_{n}} \int_{-\infty}^{\infty}\left\{\left\{F_{i}, H^{1}\right\}\right\} d t, \quad i=1, \ldots, m .
$$

It would be of interest to apply such a generalization to the Toda lattice and related completely integrable systems.

\section{§5. An Example Based on the Rigid Body with Attachments}

In this section we consider a model problem based on the rigid body in the absence of gravity with attachments which spin freely about axes coincident with one of the bodies' principal axes (Figure 4). The full Hamiltonian for this problem is quite complicated and involves cross coupling terms. These terms lead to a major modification in the "unperturbed" homoclinic orbits, which still exist for the integrable case, occurring when the attachment preserves $S^{1}$ symmetry about 
its axis. It is therefore necessary to recompute these homoclinic orbits. Moreover, the presence of coupling terms in the unperturbed Hamiltonian necessitates the use of the methods developed in Section 6 below. Therefore, in order to present a conceptually simple example, we will omit such terms so that our model problem has the simple product structure described in Section 4. For full details on the physical rigid body problem, see Koiller [1982]; also see Hubert [1980] for a practical example involving attitude control in spacecraft.

Our model problem has the Hamiltonian

$$
\begin{aligned}
H^{\varepsilon}= & \frac{1}{2} \sum_{j=1}^{3} m_{j}^{2} / I_{j}+I^{2} / 2 J_{1}-\frac{\varepsilon}{2}\left\{\frac{m_{1}^{2}}{I_{1}^{2}}+\frac{m_{2}^{2}}{I_{2}^{2}} \cos ^{2} \theta+\frac{m_{3}^{2}}{I_{3}^{2}} \sin ^{2} \theta+\frac{I^{2}}{J_{1}^{2}}\right\} \\
& +O\left(\varepsilon^{2}\right) \\
= & F+G+\varepsilon H^{1}+O\left(\varepsilon^{2}\right)
\end{aligned}
$$

where $I_{j}$ and $J_{j}$ are related to moments of inertia of the body and its attachment and $(\theta, I)$ play the role of action angle variables for the attachment. Since the unperturbed system is a product flow on $\left(S^{2}\right) \times\left(\mathbf{R} \times S^{1}\right)$, the product of the coadjoint orbit of $S O(3)$ with the $(I, \theta)$ cylinder, we can use (3.9) to write the homoclinic orbits for an energy level

$$
H^{0}=h=\frac{1}{2}\left(\frac{\ell^{2}}{I_{2}}+\frac{k^{2}}{J_{1}}\right),
$$

where $\ell^{2}=m_{1}^{2}+m_{2}^{2}+m_{3}^{2}$ and $k$ is a constant, as

$$
\left\{\begin{aligned}
m_{1} & = \pm \ell \sqrt{\frac{a_{1}}{-a_{2}}} \operatorname{sech}\left(-\sqrt{a_{1} a_{3}} \ell t\right) \\
m_{2} & = \pm \ell \tanh \left(-\sqrt{a_{1} a_{3}} \ell t\right) \\
m_{3} & = \pm \ell \sqrt{\frac{a_{3}}{-a_{2}}} \operatorname{sech}\left(-\sqrt{a_{1} a_{3}} \ell t\right) \\
I & =k \quad \text { (constant) } \\
\theta & =\frac{k}{J_{1}} t+\theta^{0} .
\end{aligned}\right.
$$

To show that transverse homoclinic orbits occur for $\varepsilon \neq 0$ we need only show that the Melnikov function

$$
M\left(\theta^{0}\right)=\frac{1}{\Omega(I)} \int_{-\infty}^{\infty}\left\{\left\{F, H^{1}\right\}\right\} d t,
$$

has simple zeros, all other conditions of Theorem 4.3 being immediately satisfied. Note that we must set $k>0$, so that $I>0$ for the unperturbed system and hence the inversion of $H^{\varepsilon}=h$ goes through. The Lie-Poisson bracket is given by (3.6): 


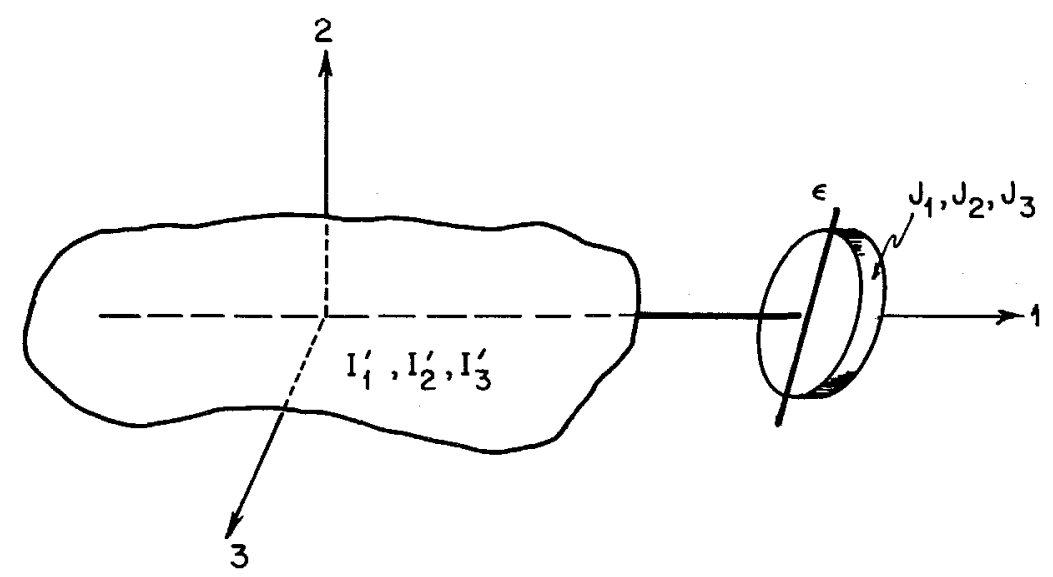

FIGURE 4.

$$
\begin{aligned}
\left\{\left\{F(m), H^{1}\left(m, I, \theta-\theta^{0}\right)\right\}\right\} & =-m \cdot \nabla_{m} F \times \nabla_{m} H^{1} \\
& =\left(\frac{a_{1}}{I_{1}^{2}}+\frac{a_{2} \cos ^{2} \theta}{I_{2}^{2}}+\frac{a_{3} \sin ^{2} \theta}{I_{3}^{2}}\right) m_{1} m_{2} m_{3} .
\end{aligned}
$$

Noting that on any homoclinic orbit, $m_{1}$ and $m_{3}$ are even while $m_{2}$ is odd, it follows that the constant term $\left(a_{1} / I_{1}^{2}\right) m_{1} m_{2} m_{3}$ vanishes in the Melnikov integral and we are left with

$$
\begin{aligned}
M\left(\theta^{0}\right) & =\frac{1}{\left(k / J_{1}\right)} \int_{-\infty}^{\infty} m_{1} m_{2} m_{3}\left(\frac{a_{2}}{I_{2}^{2}} \cos ^{2} \theta+\frac{a_{3}}{I_{3}^{2}} \sin ^{2} \theta\right) d \theta, \\
& =\frac{J_{1}}{2 k} \int_{-\infty}^{\infty} m_{1} m_{2} m_{3}\left(\frac{a_{2}}{I_{2}^{2}} \cos 2 \theta-\frac{a_{3}}{I_{3}^{2}} \cos 2 \theta\right) d \theta, \\
& =\frac{J_{1}}{2 k}\left(\frac{a_{2}}{I_{2}^{2}}-\frac{a_{3}}{I_{3}^{2}}\right) \int_{-\infty}^{\infty} m_{1} m_{2} m_{3} \cos 2 \theta d \theta .
\end{aligned}
$$

Inserting the expressions for the homoclinic orbits, we get

$$
\begin{aligned}
M\left(\theta^{0}\right) & =\frac{J_{1}}{2 k}\left(\frac{a_{2}}{I_{2}^{2}}-\frac{a_{3}}{I_{3}^{2}}\right) \int_{-\infty}^{\infty}\left(\frac{\ell^{3} \sqrt{a_{1} a_{3}}}{-a_{2}}\right) \operatorname{sech}^{2}\left(-\sqrt{a_{1} a_{3}} \ell t\right) \\
& \tanh \left(-\sqrt{a_{1} a_{3}} \ell t\right) \cos 2\left(\frac{k}{J_{1}} t+\theta^{\circ}\right) d t \\
& =C\left[\int_{-\infty}^{\infty} \operatorname{sech}^{2}\left(-\sqrt{a_{1} a_{3}} \ell t\right) \tanh \left(-\sqrt{a_{1} a_{3}} \ell t\right) \sin \left(\frac{2 k t}{J_{1}}\right) d t\right] \sin 2 \theta^{\circ}
\end{aligned}
$$

where

$$
C=\frac{J_{1}}{2 k}\left(\frac{a_{2}}{I_{2}^{2}}-\frac{a_{3}}{I_{3}^{2}}\right) \frac{\ell^{3} \sqrt{a_{1} a_{3}}}{a_{2}} \neq 0 .
$$


The integral of (5.6) may be evaluated by the method of residues to obtain

$$
M\left(\theta^{0}\right)=C \cdot \frac{2 \pi k^{2}}{J_{1}^{2}\left(-\sqrt{a_{1} a_{3} \ell}\right)^{3}} \operatorname{cosech}\left(\frac{k \pi}{-\sqrt{a_{1} a_{3} \ell} J_{1}}\right) \sin 2 \theta^{0},
$$

which has simple zeros. We therefore have

5.1 Theorem. The simplified model for a free rigid body with a single slightly asymmetrical freely rotating attachment on one of its principal axes possesses transverse heteroclinic orbits and hence Smale horseshoes in a suitably chosen cross section of the constant energy surface with $k>0$.

This implies that the rigid body equations with an additional attachment are nonintegrable. More precisely, if we make a Markov partition of the invariant sphere consisting of the four open regions filled with periodic motions in the unperturbed case (Figure 1), then the dynamics of the perturbed Poincare map are conjugate to the subshift of finite type on these four symbols. To see this we sketch the homoclinic structure on the sphere in Figure 5, identifying one of the centers (in region A) with the point at infinity. It is clear that orbits starting near the manifold on the 'boundary' of regions 2 and 3 can be selected such that they pass either from region $2 \rightarrow 2$ or $2 \rightarrow 3$ or $3 \rightarrow 2$ or $3 \rightarrow 3$. Similarly on the border of 1,2 orbits can be found passing from $1 \rightarrow 1,1 \rightarrow 2,2 \rightarrow 1$ or $2 \rightarrow 2$. Continuing in this way we find the transition matrix.

$$
A=\left[a_{i j}\right]=\left[\begin{array}{llll}
1 & 1 & 0 & 1 \\
1 & 1 & 1 & 0 \\
0 & 1 & 1 & 1 \\
1 & 0 & 1 & 1
\end{array}\right]
$$

where $a_{i j}=1$ if there is an orbit from region $i$ to region $j$ and $a_{i j}=0$ if there is no such orbit.

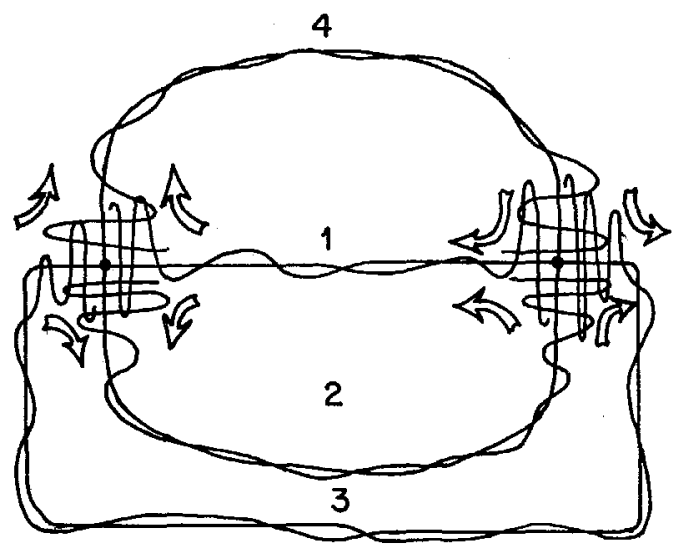

FIGURE 5. The homoclinic structure of the perturbed manifold $W^{s}(0, \pm \ell, 0), W^{u}(0, \pm \ell, 0)$ on the sphere. 
If two or more attachments are added to the free rigid body then Arnold diffusion may take place. Taking a system with two identical attachments on axis 1 , each free to rotate independently, with momenta $I, J$ and angles $\theta, \phi$, we have the Hamiltonian

$$
\begin{aligned}
H^{\varepsilon}= & F+G_{1}+G_{2}+\varepsilon H^{1}+O\left(\varepsilon^{2}\right) \\
= & \frac{1}{2}\left(\sum_{j=1}^{3} \frac{m_{j}^{2}}{I_{j}}+\frac{I^{2}+J^{2}}{J_{1}}\right) \\
& -\frac{\varepsilon}{2}\left\{\frac{m_{1}^{2}}{I_{1}^{2}}+\frac{m_{2}^{2}}{I_{2}^{2}}\left(\cos ^{2} \theta+\cos ^{2} \phi\right)+\frac{m_{3}^{2}}{I_{3}^{2}}\left(\sin ^{2} \theta+\sin ^{2} \phi\right)+\frac{I^{2}+J^{2}}{J_{1}^{2}}\right\} \\
& +O\left(\varepsilon^{2}\right) .
\end{aligned}
$$

In this case the Melnikov vector consists of the pair of functions

$$
\begin{aligned}
& M_{1}\left(\theta^{0}, \phi^{0}\right)=\int_{-\infty}^{\infty}\left\{I, H^{1}\right\} d t \\
& M_{2}\left(\theta^{0}, \phi^{0}\right)=\int_{-\infty}^{\infty} \frac{1}{\Omega_{2}(k)}\left\{\left\{F, H^{1}\right\}\right\},
\end{aligned}
$$

where $\left\{I, H^{1}\right\}=-\partial H^{1} / \partial \theta$ is the usual canonical Poisson bracket and $\Omega_{2}=J / J_{2}$. Computations similar to those above (cf. Holmes and Marsden [1982b, §4]) show that

$$
\begin{aligned}
& M_{1}=C_{1} \sin 2 \theta^{\circ} \\
& M_{2}=C_{2} \sin 2 \theta^{\circ}+C_{3} \sin 2 \phi^{0}
\end{aligned}
$$

where the $C_{j}$ are nonzero constants depending on $I, J, J_{1}$, and the choice of total energy and energy in each 'mode' $\left(E=\ell^{2} / 2 I_{2}+k_{1}^{2} / 2 J_{1}+k_{2}^{2} / 2 J_{1}\right)$. Thus $M$ has simple (transverse) zeros for $\theta^{0}=m \pi, \phi^{0}=n \pi, m, n \in \mathbf{Z}$. Since $\Omega_{1}^{\prime}(I)=$ $1 / J_{1} \neq 0$, all the conditions (H1)-(H4) of Theorem 4.4 hold. Therefore, simplified model for the rigid body with two attachments as specified exhibits Arnold diffusion. The existence of a transition chain of two-tori connected by heteroclinic orbits, (see Holmes and Marsden [1982b, §3] for a discussion) implies that angular momentum can be transferred back and forth between the two spinning attachments in a chaotic manner.

Remark. An amusing corollary for the case of a single attachment is that there are configurations of the Euler elastica for which the sequence of loops above and below the mean level can be prescribed in advance. (For example the loops can be coded by the binary expansion of an irrational number.) This follows from the above calculations as modified by Koiller [1982], and the remarkable fact that the elastica equations have the form of the equations of a rigid body with an attachment; see Love [1927, page 400]. 


\section{§6. Melnikov Theory for Systems with $S^{\mathbf{1}}$ Symmetry}

We now develop a version of the Melnikov theory that applies to perturbations of a two degree of freedom system with an $S^{1}$ symmetry. We have chosen this context with applications to the motion of a nearly symmetric heavy top in mind.

The key new feature is that the unperturbed system is no longer assumed to be a product system consisting of variables with a homoclinic orbit and action angle variables. Rather, this product structure is generalized to the assumption of an $S^{1}$ reduction.

Roughly speaking, our unperturbed Hamiltonian $H^{0}$ no longer can be split as

$$
H^{0}(q, p, I)=F(q, p)+G(I)
$$

so that the frequency function $\Omega=\partial H^{0} / \partial I$ now may depend on $(p, q)$. This is in fact the situation for the nearly symmetric heavy top.

Let us start with a four-dimensional symplectic manifold $P$, whose points are denoted $x$. Suppose $S^{1}$ acts on $P$ by canonical transformations and has an $\mathrm{Ad}^{*-}$ equivariant momentum map $J: P \rightarrow \mathbf{R}$. Let the reduced space be denoted

$$
P_{\mu}=J^{-1}(\mu) / S^{1}
$$

(see Marsden and Weinstein [1974]).

For the heavy top, $P=T^{*} S^{2}$ and $S^{1}$ consists of rotations about the axis of symmetry. To keep the notation consistent, we shall use $\psi$ for the angle on $S^{1}$. Motivated by this example, we allow $P_{\mu}$ to have isolated singularities, but in this case we demand that the constructions carried out below make sense at the singular points. For the heavy top this causes no difficulties.

Let points in the reduced space be denoted $u \in P_{\mu}$. Thus, $u$ consists of an $S^{1}$ orbit in $P$, lying in the level set $J^{-1}(\mu)$. Choosing a slice (cross section) for this action, $\psi$ parametrizes the point on the orbit and of course the value of $J$ labels the surface $J^{-1}(\mu)$. Thus, we write points $x$ as

$$
x=(u, \psi, J) \text {. }
$$

See Figure 6.

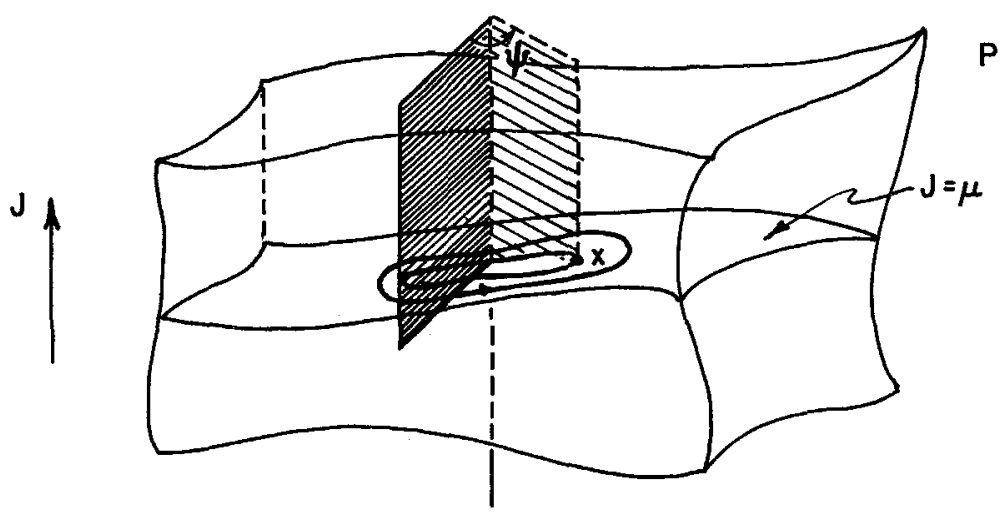

FIGURE 6. 
In this notation, a function of $x$ is $S^{1}$ invariant if and only if it can be expressed as a function of $u$ and $J$ alone. Such functions induce functions on $P_{\mu}$, the reduced space, and have well-defined Poisson brackets on $P_{\mu}$. In fact, the Poisson bracket of two such functions is just that function induced on $P_{\mu}$ by their Poisson bracket on $P$. Of course any $S^{1}$ invariant Hamiltonian on $P$ gives a completely integrable system, the integrals being $J$ and $H$, or equivalently $H$ for the reduced one degree of freedom system.

Now assume that we start with an $S^{1}$ invariant Hamiltonian $H^{0}(u, J)$ and we perturb it by a general Hamiltonian. Thus, write

$$
H^{\varepsilon}(u, \psi, J)=H^{0}(u, J)+\varepsilon H^{1}(u, \psi, J)+O\left(\varepsilon^{2}\right) .
$$

Now we must generalize the procedure of Holmes and Marsden [1982a] to this context.

We begin by letting

$$
\Omega(u, J)=\frac{\partial H^{0}}{\partial J}(u, J),
$$

and assuming $\Omega$ is positive, so that $H^{0}(u, J)$ is invertible in the $J$-variable. For $\varepsilon$ small

$$
H^{\varepsilon}(u, \psi, J)=h
$$

can be solved for $J$. Write $H_{u}^{0}(J)=H^{0}(u, J)$ and $\left(H_{u}^{0}\right)^{-1}$ for its inverse in the $J$ variable.

6.1 Proposition. $J=L^{\varepsilon}(u, \psi, h)=L^{0}(u, h)+\varepsilon L^{1}(u, \psi, h)+O\left(\varepsilon^{2}\right)$ where

$$
L^{0}(u, h)=\left(H_{u}^{0}\right)^{-1}(h),
$$

and

$$
L^{1}(u, \psi, h)=\frac{-H^{1}\left(u, \psi, L^{0}(u, h)\right)}{\Omega\left(u, L^{0}(u, h)\right)} .
$$

Proof. (6.4) reads

$$
H^{\varepsilon}\left(u, \psi, L^{0}+\varepsilon L^{1}+O\left(\varepsilon^{2}\right)\right)=h,
$$

i.e.

$$
H^{0}\left(u, L^{0}+\varepsilon L^{1}+O\left(\varepsilon^{2}\right)\right)+\varepsilon H^{1}\left(u, \psi, L^{0}+\varepsilon L^{1}+O\left(\varepsilon^{2}\right)\right)=h
$$

i.e.

$$
H^{0}\left(u, L^{0}\right)+\varepsilon \Omega\left(u, L^{0}\right)+\varepsilon H^{1}(u, \psi, h)=h+O\left(\varepsilon^{2}\right) .
$$

Comparing powers of $\varepsilon$ gives the result.

As in Marsden [1981, Lecture 4] we can arrange things so that $\psi$ and $J$ are conjugate variables. Thus, under the dynamics of $H^{\varepsilon}$, we have 


$$
\dot{\psi}=\left\{\psi, H^{\varepsilon}\right\}=\frac{\partial H^{\varepsilon}}{\partial J}
$$

Now we change time variables from $t$ to $\psi$ and write ' for $d / d \psi$. Thus, for a given function $F(u)$,

$$
F^{\prime}=\frac{\dot{F}}{\dot{\psi}}=\left\{F, H^{\varepsilon}\right\} / \frac{\partial H^{\varepsilon}}{\partial I} .
$$

However, from (6.4) and properties of the Poisson bracket,

$$
\left\{F(u), H^{\varepsilon}\left(u, \psi, L^{\varepsilon}(u, \psi, h)\right)\right\}=0
$$

so

$$
\left\{F, H^{\varepsilon}\right\}+\frac{\partial H^{\varepsilon}}{\partial I}\left\{F, L^{\varepsilon}\right\}=0 .
$$

Comparing (6.8) and (6.9), results in

$$
F^{\prime}=-\left\{F, L^{\varepsilon}\right\}=-\left\{F, L^{0}\right\}-\varepsilon\left\{F, L^{1}\right\}+O\left(\varepsilon^{2}\right) .
$$

Thus, $(6.10)$ is in the form of a periodically forced Hamiltonian system on the reduced phase space. The brackets in (6.10) are taken in the $u$-variable alone. Thus, we have:

6.2 Proposition. The evolution of $u$ as a function of $\psi$ is a $\psi$-dependent Hamiltonian system in the reduced phase space. The equations of evolution are given by $(6.10)$.

Now suppose that the reduced system for $\varepsilon=0$ has a homoclinic orbit. To detect the presence of transverse homoclinic orbits for $\varepsilon \neq 0$ we must integrate $\left\{L^{0}, L^{1}\right\}$ around this orbit. In examples, $L^{0}$ and $L^{1}$ and their bracket can be laborious to compute. It will save some effort if we relate $\left\{L^{0}, L^{1}\right\}$ to $H^{0}$ and $H^{1}$.

\subsection{Proposition.}

$$
\left\{L^{0}, L^{1}\right\}=\left(\frac{1}{\Omega}\right)\left\{H^{0}, \frac{H^{1}}{\Omega}\right\}
$$

where $\Omega$ is evaluated at $u, J$ and the brackets are taken with respect to $u$, holding $\psi, J$ fixed.

Remarks 1. Formula (6.11) is a generalization of (3.1) of Holmes and Marsden [1982a]. In the latter case $\Omega$ was independent of $u$.

2. The above development does not depend on the dimension of $P$.

Proof of 6.3. Given $F(u)$, we first compute $\left\{L^{0}, F\right\}$. From (6.5), $H^{0}\left(u, L^{0}(u, h)\right)=h$, so

$$
\left\{H^{0}, F\right\}+\Omega\left\{L^{0}, F\right\}=0 .
$$


Thus

$$
\left\{L^{0}, F\right\}=-\frac{1}{\Omega}\left\{H^{0}, F\right\} .
$$

Next, from (6.6) we obtain

$$
\left\{G, L^{1}\right\}=-\left\{G, \frac{H^{1}}{\Omega}\right\}-\frac{1}{\Omega} \frac{\partial H^{1}}{\partial J}\left\{G, L^{0}\right\}+\frac{H^{1}}{\Omega^{2}} \frac{\partial \Omega}{\partial J}\left\{G, L^{0}\right\} .
$$

Taking $G=L^{0}$ in (6.13), the last two terms vanish, so

$$
\left\{L^{0}, L^{1}\right\}=-\left\{L^{0}, \frac{H^{1}}{\Omega}\right\} .
$$

By (6.12) this gives the stated result.

Assembling these results as we did in Section 4, we obtain the following.

6.4. Theorem. Consider a two degree of freedom Hamiltonian system on a symplectic manifold $P$ with an $S^{1}$ symmetry and a Hamiltonian of the form (6.2). Assume that $J$ is chosen and fixed so that the reduced system $H^{0}(u, J)$ has a homoclinic (or heteroclinic) orbit $\bar{u}(t)$ in the reduced space $P_{\mu}$ and so $\Omega(\bar{u}(t), J)>0$. Let

$$
\psi(t)=\int_{0}^{t} \Omega(\bar{u}(t), J) d t+\psi^{0}
$$

and let $\left\{H^{0}, H^{1} / \Omega\right\}\left(t, \psi^{0}\right)$ denote the $u$-Poisson bracket evaluated at $\bar{u}(t), \psi(t)$ and J. Let

$$
M\left(\psi^{0}\right)=\int_{-\infty}^{\infty}\left\{H^{0}, \frac{H^{1}}{\Omega}\right\}\left(t, \psi^{0}\right) d t
$$

and assume $M\left(\psi^{0}\right)$ has simple zeros as a function of $\psi^{0}$. Then for $\varepsilon$ sufficiently small, the system (6.2) has transverse homoclinic (or heteroclinic) orbits, and hence Smale horseshoes on the energy surface $H^{\varepsilon}=h$, where $h=H^{0}(\bar{u}, J)$.

6.5 Example. A simple introductory example in which $\Omega$ is not constant on the homoclinic orbit is provided by a slightly asymmetric central force problem

$$
\begin{aligned}
H^{\varepsilon}\left(r, \theta, p_{r}, p_{\theta}\right) & =\frac{p_{r}^{2}}{2}+V(r)+\frac{p_{\theta}^{2}}{2 r^{2}}+\varepsilon \sin m \theta \\
& \stackrel{\text { def }}{=} H^{0}\left(r, p_{r}, p_{\theta}\right)+\varepsilon H^{1}\left(r, \theta, p_{\theta}, p_{r}\right) .
\end{aligned}
$$

Here $r, \theta$ are the usual planar polar coordinates and $V(r)$ is a potential function with a single maximum, so that, for suitable values of $p_{\theta} \neq 0$ the effective potential $V(r)+p_{\theta}^{2} / 2 r^{2}$ has a minimum at $r_{-}$and a maximum at $r_{+}$with $r_{-}<$ 
$r_{+}$and $V(r)+p_{\theta}^{2} / 2 r^{2} \rightarrow \infty$ as $r \rightarrow 0$. Thus $H^{0}$ has a homoclinic orbit $\left(\bar{r}(t), \bar{p}_{r}(t), \theta(t)+\theta_{0}, \bar{p}_{\theta}\right)$, where $\bar{r}(t) \rightarrow r_{-}, \bar{p}_{r}(t) \rightarrow 0$ as $t \rightarrow \pm \infty, \bar{r}(0)<r_{-}$, $\bar{p}_{r}(0)=0, J=\bar{p}_{\theta}=$ constant $\neq 0$ and

$$
\bar{\theta}(t)=\int_{0}^{t} \Omega(t) d t ; \quad \Omega=p_{\theta} / \bar{r}^{2}(t)
$$

where $\Omega=\partial H^{0} / \partial p_{\theta}$ is evaluated on the homoclinic orbit. We therefore have

$$
\begin{aligned}
\left\{H^{0}, H^{1} / \Omega\right\} & =\frac{\partial H^{0}}{\partial r} \frac{\partial}{\partial p_{r}}\left(\frac{H^{1}}{\Omega}\right)-\frac{\partial H^{0}}{\partial p_{r}} \frac{\partial}{\partial r}\left(\frac{H^{1}}{\Omega}\right) \\
& =-p_{r}\left(\frac{2 r}{p_{\theta}} \sin m \theta\right),
\end{aligned}
$$

and so the Melnikov function obtained by integrating around the homoclinic orbit is

$$
\begin{aligned}
M\left(\theta_{0}\right)= & -\frac{2}{\bar{p}_{\theta}} \int_{-\infty}^{\infty} \bar{r}(t) \bar{p}_{r}(t) \sin m\left(\bar{\theta}(t)+\theta_{0}\right) d t \\
= & -\frac{2}{\bar{p}_{\theta}}\left[\int_{-\infty}^{\infty} \bar{r}(t) \bar{p}_{r}(t) \sin m \bar{\theta}(t) d t \cdot \cos m \theta_{0}\right. \\
& \left.+\int_{-\infty}^{\infty} \bar{r}(t) \bar{p}_{r}(t) \cos m \bar{\theta}(t) d t \cdot \sin m \theta_{0}\right] .
\end{aligned}
$$

Since $\bar{r}$ is even and $\bar{p}_{r}, \bar{\theta}$ are odd, the second integral is identically zero and we obtain

$$
M\left(\theta_{0}\right)=A \cos m \theta_{0},
$$

which has simple zeros if $A=-\left(2 / \bar{p}_{\theta}\right) \int_{-\infty}^{\infty} \bar{r}(t) \bar{p}_{r}(t) \sin m \bar{\theta}(t) d t$ is nonzero. Almost all choices of $V(r)$ will satisfy this condition, and hence the asymmetric problem will be nonintegrable.

We note that this nonintegrability arises from two factors: the presence of the coupled frequency term $p_{\theta}^{2} / 2 r^{2}$ in the unperturbed Hamiltonian, and the angular dependence of the perturbation, $\sin m \theta$. Models similar to this have been used in the description of barred and spiral galaxies, in which nonintegrability and the presence of 'ergodic' orbits is related to the escape of stars from the system (cf. Contopoulos [1981] and references therein).

\section{\$7. Example: The Motion of a Nearly Symmetric Heavy Top}

We now show that Theorem 6.4 implies:

7.1 Theorem. If $I_{1} / I_{3}$ is sufficiently large, $I_{2}=I_{1}+\varepsilon$ and $\varepsilon$ is sufficiently small, $\varepsilon \neq 0$, then the Hamiltonian system for heavy top (see 3.11 and 3.12) has 
transverse homoclinic orbits in the Poincare map for the $\psi$-variable on energy surfaces close to the homoclinic orbit described in 3.2 or 3.4 .

7.2 Corollary. The heavy top close to the symmetric top has no analytic integrals other than the energy and angular momentum about the vertical axis.

Remarks 1. As we have already discussed, this corollary has recently been obtained by Ziglin [1980], but by rather different methods. Moreover, our result 7.1 shows the existence of 'chaotic' orbits.

2. $I_{1} / I_{3}$ being large can be replaced by $0<\gamma<1$ and the integral 7.13 below being nonzero. This integral is nonzero for most values of $M, \beta, I_{1}, I_{3}$, as we shall show.

We shall prove Theorem 7.1 in the Euler angle representation first and then sketch how the proof can be alternatively obtained using the Lie-Poisson description.

In (3.12) let $I_{2}=I_{1}+\varepsilon$. This gives

$$
H^{0}=\frac{1}{2}\left\{\frac{\left(p_{\phi}-p_{\psi} \cos \theta\right)^{2}}{I_{1} \sin ^{2} \theta}+\frac{p_{\theta}^{2}}{I_{1}}+\frac{p_{\psi}^{2}}{I_{3}}\right\}+M g \ell \cos \theta
$$

and

$$
H^{1}=-\frac{1}{2 I_{1}^{2} \sin ^{2} \theta}\left(\left(p_{\phi}-p_{\psi} \cos \theta\right) \cos \psi-p_{\theta} \sin \theta \sin \psi\right)^{2} .
$$

Note that $H^{0}$ is the Hamiltonian for the symmetric top and so has a homoclinic orbit given by (3.15).

Since $\psi$ and $p_{\psi}$ are conjugate variables and $J=p_{\psi}$, we have from (6.3),

$$
\Omega=\frac{p_{\psi}}{I_{3}}-\left(\frac{p_{\phi}-p_{\psi} \cos \theta}{I_{1} \sin ^{2} \theta}\right) \cdot \cos \theta .
$$

On the homoclinic orbit, $p_{\phi}=p_{\psi}=$ constant $\equiv I_{1} b$, so (7.3) becomes

$$
\Omega=b\left(\frac{I_{1}}{I_{3}}-\frac{\cos \theta}{1+\cos \theta}\right) \text {. }
$$

7.3. Lemma. With $p_{\phi}=p_{\psi}=I_{1} b, \beta=2 \mathrm{Mg} \ell / I_{1}$,

$$
\dot{\phi}=\frac{b}{1+\cos \theta} \text { and } \dot{\theta}=\frac{p_{\theta}}{I_{1}}
$$

we have

$$
\left\{H^{0}, \frac{H^{1}}{\Omega}\right\}_{\theta, p_{\theta}}=\frac{1}{\Omega(\theta)}(A(\theta) \sin 2 \psi+B(\theta) \cos 2 \psi+C(\theta))
$$


where

and

$$
\begin{aligned}
& A(\theta)=\frac{b}{2}\left(\dot{\phi}^{2}-\frac{\beta}{2}+\frac{\dot{\theta}^{2} \dot{\phi}^{2}}{b \Omega}\right)(1-\cos \theta)-\dot{\theta}^{2} \\
& B(\theta)=\left(\dot{\theta} \dot{\phi}^{2}-\frac{\beta \dot{\theta}}{4}+\frac{\dot{\theta}^{3} \dot{\phi}^{2}}{4 b \Omega}\right) \sin \theta-\frac{\dot{\theta} \dot{\phi}^{4}}{4 b \Omega} \sin ^{3} \theta \\
& C(\theta)=\dot{\theta}\left(\frac{\beta}{4}-\frac{\dot{\phi}^{4} \sin ^{2} \theta+\dot{\theta}^{2} \dot{\phi}^{2}}{4 b \Omega}\right) \sin \theta .
\end{aligned}
$$

Proof. The computations are slightly tedious but straightforward. We write

$$
\begin{aligned}
\left\{H^{0}, \frac{H^{1}}{\Omega}\right\} & =\frac{1}{\Omega}\left\{H^{0}, H^{1}\right\}-\frac{H^{1}}{\Omega^{2}}\left\{H^{0}, \Omega\right\}, \\
\left\{H^{0}, H^{1}\right\} & =\frac{\partial H^{0}}{\partial \theta} \frac{\partial H^{1}}{\partial p_{\theta}}-\frac{\partial H^{0}}{\partial p_{\theta}} \frac{\partial H^{1}}{\partial \theta}, \\
\left\{H^{0}, \Omega\right\} & =-\frac{\partial H^{0}}{\partial p_{\theta}} \frac{\partial \Omega}{\partial \theta},
\end{aligned}
$$

and compute that

$$
\begin{aligned}
& \frac{\partial H^{0}}{\partial \theta}=-I_{1}\left(\dot{\phi}^{2} \sin \theta-\frac{\beta}{2} \sin \theta\right), \\
& \frac{\partial H^{0}}{\partial p_{\theta}}=\dot{\theta} \\
& \frac{\partial H^{1}}{\partial \theta}=-\dot{\phi}^{2} \sin \theta \cos ^{2} \psi+\frac{\dot{\phi} \dot{\theta}}{2} \sin 2 \psi, \\
& \frac{\partial H^{1}}{\partial p_{\theta}}=\frac{1}{I_{1}}\left(\frac{\dot{\phi}}{2} \sin \theta \sin 2 \psi-\dot{\theta} \sin ^{2} \psi\right), \\
&\left\{H^{0}, H^{1}\right\}=\frac{\dot{\phi}}{2}\left[\left(\dot{\phi}^{2}-\frac{\beta}{2}\right) \sin ^{2} \theta-\dot{\theta}^{2}\right] \sin 2 \psi \\
&+\dot{\theta} \dot{\phi}^{2} \sin \theta \cos 2 \psi-\frac{\beta \dot{\theta}}{4} \sin \theta(\cos 2 \psi-1), \\
& \frac{\partial \Omega}{\partial \theta}=\frac{\dot{\phi}^{2}}{b} \sin \theta,
\end{aligned}
$$

and

$$
\left\{H^{0}, \Omega\right\}=-\frac{\dot{\theta} \dot{\phi}^{2}}{b} \sin \theta
$$


Substituting these expressions into (7.6) and simplifying yields (7.5).

The Melnikov function is given by

$$
M\left(\psi^{0}\right)=\int_{-\infty}^{\infty}\left\{H^{0}, \frac{H^{1}}{\Omega}\right\} d t
$$

where the integral is evaluated along the homoclinic orbit

$$
\cos \theta=1-\gamma \operatorname{sech}^{2}\left(\frac{\sqrt{\beta \gamma} t}{2}\right), \quad p_{\theta}=I_{1} \dot{\theta}
$$

(see (3.15)) and where

$$
\psi(t)=\int_{0}^{t} \Omega(t) d t+\psi^{0}=\bar{\psi}(t)+\psi^{0}
$$

and

$$
\Omega(t)=b\left(\frac{I_{1}}{I_{3}}+\frac{1-\gamma \operatorname{sech}^{2}\left(\sqrt{\frac{\beta \gamma}{2} t}\right)}{\gamma \operatorname{sech}^{2}\left(\sqrt{\frac{\beta \gamma}{2} t}\right)}\right) .
$$

Note that if $0<\gamma<1$, or if $I_{1} / I_{3}$ is sufficiently large, $\Omega(t)>0$. Substituting (7.5) and (7.9) into (7.7) yields

$$
\begin{aligned}
M\left(\psi^{0}\right)= & {\left[\int_{-\infty}^{\infty} \frac{1}{\Omega}(A(\theta) \sin 2 \bar{\psi}+B(\theta) \cos 2 \bar{\psi}) d t\right] \cos 2 \psi^{0} } \\
& +\left[\int_{-\infty}^{\infty} \frac{1}{\Omega}(A(\theta) \cos 2 \bar{\psi}-B(\theta) \sin 2 \bar{\psi}) d t\right] \sin 2 \psi^{0} \\
& +\int_{-\infty}^{\infty} \frac{1}{\Omega} C(\theta) d t
\end{aligned}
$$

The first, second and fifth terms are odd functions of $t$ and so we obtain the following

\subsection{Lemma.}

$$
M\left(\psi^{0}\right)=\left[\int_{-\infty}^{\infty} \frac{1}{\Omega(\theta)}(A(\theta) \cos 2 \bar{\psi}-B(\theta) \sin 2 \bar{\psi}) d t\right] \sin 2 \psi^{0}
$$

Now we observe that at $t=0, A=b^{3} \gamma^{2} / 4(2-\gamma)>0$ and $B=0$. It follows that for $I_{1} / I_{3}$ sufficiently large, the portion of the integral from the first term near $t=0$ dominates and so the integral must be a nonzero number. Thus $M\left(\psi^{0}\right)$ has simple zeros and the theorem is proved. 
Remark. For any given $b, \beta, I_{1}, I_{3}$ (with $\gamma=2-b^{2} / \beta$ and $b^{2}<2 \beta, b, \beta>$ 0 ) one needs only the condition that

$$
\int_{-\infty}^{\infty} \frac{1}{\Omega}(A(\theta) \cos 2 \bar{\psi}-B(\theta) \sin 2 \bar{\psi}) d t
$$

be nonzero. Since we cannot evaluate (7.13) analytically, it does not seem so simple to decide exactly when (7.13) vanishes. However, since it is nonzero for $I_{1} / I_{3}$ large, it follows that (7.13) can vanish for at most a finite set of values of $b, \beta, I_{1}, I_{3}$ as it is analytic. Thus, we can be sure of transverse homoclinic orbits for generic $M, I_{1}, I_{3}, b$, if $0<\gamma<1$. [See Kopell and Washburn [1982] for a related use of analyticity.]

Finally, we indicate how the same computations can be done using the LiePoisson formalism. This actually makes the computations slightly easier, but the final result is the same.

Again, letting $I_{2}=I_{1}+\varepsilon$ in $H=(1 / 2) \sum_{j=1}^{3} m_{j}^{2} / I_{j}+M g \ell v_{3}$, we have

$$
H^{0}=\frac{m_{1}^{2}+m_{2}^{2}}{2 I_{1}}+\frac{m_{3}^{2}}{2 I_{3}}+M g \ell \mathrm{v}_{3}
$$

and

$$
H^{1}=-\frac{m_{2}^{2}}{2 I_{1}^{2}} .
$$

Next, observe that $\psi$ and $m_{3}$ are conjugate variables, where $\tan \psi=\mathrm{v}_{1} / \mathrm{v}_{2}$, so if $m \cdot \mathrm{v}=I_{1} b=m_{3}$, we have

$$
\Omega=\left\{\left\{\psi, H^{0}\right\}\right\}=b\left(\frac{I_{1}}{I_{3}}-\frac{\mathrm{v}_{3}}{1+\mathrm{v}_{3}}\right)
$$

which agrees with (7.4). To compute $\left\{\left\{H^{0}, H^{1} / \Omega\right\}\right\}$ we write (see Lemma 3.3) $H^{1}$ as a function of the reduced variables $\mathrm{v}_{2} m_{1}-\mathrm{v}_{1} m_{2}$ and $\mathrm{v}_{3}$ and compute the bracket holding $\psi$ and $m_{3}$ fixed. This is done by writing

$$
m_{2}^{2}=\frac{1}{1-\mathrm{v}_{3}^{2}}\left[\left(m \cdot \mathrm{v}-m_{3} \mathrm{v}_{3}\right) \cos \psi-\left(m_{1} \mathrm{v}_{2}-m_{2} \mathrm{v}_{1}\right) \sin \psi\right]^{2} .
$$

Setting $m \cdot \mathrm{v}=m_{3}=I_{1} b$ and using this expression to evaluate $\nabla_{m} H^{1}, \nabla_{\mathrm{v}} H^{1}$ in the Lie-Poisson brackets (3.16) we can compute $\left\{\left\{H^{0}, H^{1} / \Omega\right\}\right\}$. (Note that, since $H^{0}$ is $S^{1}$ invariant we need not substitute for $m_{1}$ or $m_{2}$ in that expression.) Finally, using Table 1 we can express the Lie-Poisson bracket in terms of the Euler angles and obtain precisely the expression (7.11) derived earlier. Then the proof may be completed as it was using Euler angles.

\section{REFERENCES}

R. Abraham \& J. MARSDen [1978], Foundations of Mechanics, 2nd edition, Addison-Wesley, Reading, MA. 
L. D. Akulenko, D. D. Leshchenko \& F. L. Chernouśko [1979], Perturbed motions of a rigid body close to the Lagrange case, PMM 43, (771-778).

J. ARMs, J. MARSDEN \& V. MONCRIEF [1981], Symmetry and bifurcations of momentum mappings, Comm. Math. Phys. 78, (455-478).

V. I. ARNOLD [1964], Instability of dynamical systems with several degrees of freedom, Dokl. Akad. Nauk. SSSR 156, (9-12).

V. I. ARNOLD [1966], Sur la géometrie differentielle des groupes de Lie de dimension infinie et ses applications a l'hydrodynamique des fluids parfaits, Ann. Inst. Fourier (Grenoble) 16, (319. $361)$.

V. ARNOLD [1978], Mathematical methods of classical mechanics, Springer Graduate Texts in Math. No. 60, Springer-Verlag, New York.

G. Contopoulos [1981], The effect of resonances near corotation in barred galaxies, Astron. Astro. Phys. 102, (265-278).

D. EBIN \& J. MARSDEN [1970], Groups of diffeomorphisms and the motion of an incompressible fluid, Ann. of Math. 92, (102-163).

L. Galgoni, A. Giorgilli \& J. M. Strelcyn [1981], Chaotic motions and transition to stochasticity in the classical problem of the heavy rigid body with a fixed point, Nuovo Cimento 61, (120).

H. GoLDSTEIN [1980], Classical Mechanics, 2nd edition, Addison-Wesley, Reading, MA.

B. GREENSPAN \& P. J. Holmes [1981], Homoclinic orbits, subharmonics and global bifurcations in forced oscillations, in "Non-linear Dynamics and Turbulence," (G. Barenblatt, G. Iooss \& D. D. Joseph, Eds.), Pitman, London.

V. Guillemin \& S. STERnBerg [1980], The moment map and collective motion, Ann. Physics 127, $(220-253)$.

V. GullLemin \& S. STERNBerg [1982], (in preparation).

P. HoLMES [1980], Averaging and chaotic motions in forced oscillations, SIAM J. Appl. Math. 38, $(65-80)$ and 40, (167-168).

P. J. HolmES \& J. E. MARSDEN [1981], A partial differential equation with infinitely many periodic orbits: chaotic oscillations of a forced beam, Arch. Rational Mech. Anal. 76, (135-166).

P. J. Holmes \& J. E. MARSDEN [1982a], Horseshoes in perturbations of Hamiltonian systems with two degrees of freedom, Comm. Math. Phys. 23, (523-544).

P. J. HOLMES \& J. E. MARSDEN [1982b], Melnikov's method and Arnold diffusion for perturbations of integrable Hamiltonian systems, J. Math. Phys. 23, (669-675).

C. H. HUBERT [1980], An attitude acquisition technique for dual-spin spacecraft, PhD Thesis, Cornell University.

D. KAZHDAN, B. KostANT \& S. STERNBERG [1978], Hamiltonian group actions and dynamical systems of Calogero type, Comm. Pure Appl. Math. 31, (481-508).

J. KOLLLER [1982], A mechanical system with a "wild" horseshoe, (manuscript).

N. Kopell \& R. B. WASHBURN [1982], Chaotic motions in the two degree of freedom swing equations, IEEE Trans., Nov. 1982.

M. KUMMER [1981], On the construction of the reduced phase space of a Hamiltonian system with symmetry, Indiana Univ. Math. J. 30, (281-292).

A. E. H. Love [1927], A Treatise on the Mathematical Theory of Elasticity, Cambridge University Press (reprinted by Dover, [1944]).

J. MARSDEN [1981], Lectures on Geometric Methods in Mathematical Physics, CBMS-NSF Regional Conference Series \#37, SIAM.

J. Marsden, T. Ratiu \& A. Weinstein [1982], Semi-direct products and reduction in mechanics, (preprint).

J. MARSDEN \& A. WEINSTEIN [1974], Reduction of symplectic manifolds with symmetry, Rep. Math. Phys. 5, (121-130).

J. MARSDEN \& A. WEINSTEIN [1982], The Hamiltonian structure of the Maxwell-Vlasov equations, Phys. D. 4, (394-406).

V. K. Melnikov [1963], On the stability of the center for time periodic perturbations, Trans. Moscow Math. Soc. 12, (1-57). 
T. RATIU [1981], Euler-Poisson equations on Lie algebras and the $N$-dimensional heavy rigid body, Proc. Nat. Acad. Sci. U.S.A. 78, (1327-1328).

T. RATU [1982], Euler-Poisson equations on Lie algebras and the $N$-dimensional heavy rigid body, Amer. J. Math. 104, (409-448).

T. RatIU \& P. van Moerbeke [1982], The Lagrange rigid body motion, Ann. Inst. Fourier 32, (211234).

S. L. ZIGLIN [1980], Decomposition of separatrices, branching of solutions and nonexistence of an integral in the dynamics of a rigid body, Trans. Moscow Math. Soc. 41, (287).

S. L. ZIGLIN [1981], Branching of solutions and nonexistence of integrals in Hamiltonian systems, Dokl. Akad. Nauk. SSSR 257, (26-29).

This research was partially supported by ARO Contract DAAG-29-79-C-0086 and by National Science Foundation Grants ENG 78-02891 and MCS 81-07086.

Holmes: Department of Theoretical \& Applied Mechanics, Cornell University-Ithaca, Ny 14853

Marsden: Department of Mathematics, University of California-Berkeley, CA 94720

Received July 29, 1981 